Supporting Information

\title{
Synthesis of Spongidine A, D and Petrosaspongiolide L methyl ester using Pyridine C-H Functionalization
}

Florian Bartels, Manuela Weber and Mathias Christmann* 


\section{Table of Contents}

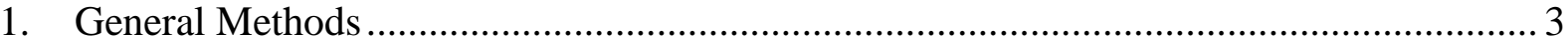

2. Scheme SI1: Proposed Mechanism of the intramolecular Minisci Reaction ...................... 4

3. Table SI1: Optimization Study for the Synthesis of Chloropyridine 4........................... 5

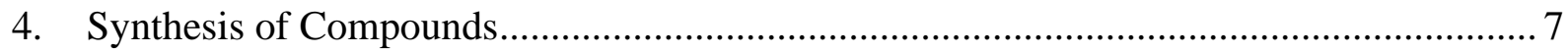

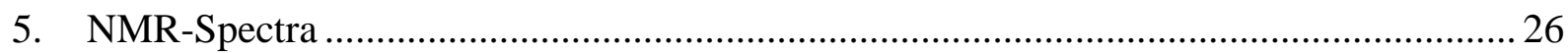

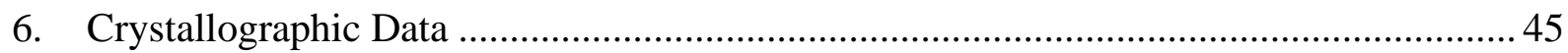

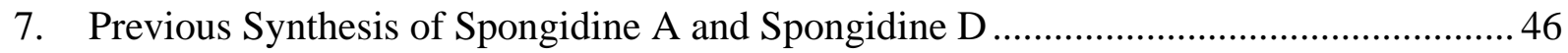

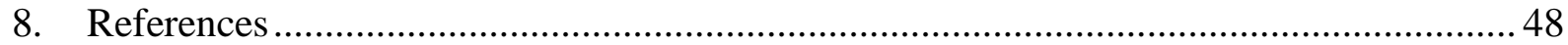




\section{General Methods}

All reactions sensitive to moisture and air were carried out using heat-gun dried $\left(630{ }^{\circ} \mathrm{C}\right)$ glassware under an argon atmosphere. Dry solvents $\left(\mathrm{CH}_{2} \mathrm{Cl}_{2}, \mathrm{Et}_{2} \mathrm{O}\right.$, THF, Toluene) were purified by Solvent Purification System M-BRAUN Glovebox Technology SPS-800. Dry DMF was obtained from Acros Organics 99.8\%, extra dry over molecular sieves. Dry pyridine was obtained from Sigma Aldrich, anhydrous, 99.8\%. Solvents for column chromatography were used after short path distillation using a rotary evaporator. Commercial reagents were used as received unless otherwise stated. Reactions were carried out under magnetic stirring with Teflon coated stirring bars and were monitored by TLC analysis on $0.20 \mathrm{~mm}$ silica gel plates (Macherey-Nagel G/UV254). Reactions were heated using an oil bath, if not stated otherwise. Visualization was performed by UV irradiation $(254 \mathrm{~nm})$ or staining of TLC plates with an acidic vanillin solution $(1 \mathrm{~g}$ vanillin, $20 \mathrm{~mL}$ conc. acetic acid, $10 \mathrm{~mL}$ conc. sulfuric acid, $170 \mathrm{~mL}$ methanol) or $\mathrm{KMnO}_{4}$ solution $\left(1.5 \mathrm{~g} \mathrm{KMnO}_{4}, 10 \mathrm{~g} \mathrm{~K}_{2} \mathrm{CO}_{3}, 1.2 \mathrm{~mL} 10 \% \mathrm{NaOH}, 200\right.$ $\left.\mathrm{mL} \mathrm{H}_{2} \mathrm{O}\right)$ and heat. Column chromatography was carried out on silica gel $60 \mathrm{M}(0.04-0.063$ $\mathrm{mm}$ ) from Macherey-Nagel. Reverse phase HPLC was carried out on Gemini-NX $5 \mu \mathrm{C} 18$, $4.6 \times 250 \mathrm{~mm}$. Concentration under reduced pressure was performed by rotary evaporation at $40{ }^{\circ} \mathrm{C}$

${ }^{1} \mathrm{H}$ NMR and ${ }^{13} \mathrm{C}$ NMR spectra were recorded on Bruker (ECP 400, AC 500, AV 700) or JEOL (ECX 400, Eclipse 500) instruments. Chemical shifts are reported relative to $\mathrm{CDCl}_{3}\left({ }^{1} \mathrm{H}\right.$ : $\left.7.26 \mathrm{ppm} ;{ }^{13} \mathrm{C}: 77.16 \mathrm{ppm}\right)$ and $\mathrm{CD}_{3} \mathrm{OD}\left({ }^{1} \mathrm{H}: 3.31 \mathrm{ppm} ;{ }^{13} \mathrm{C}: 49.00 \mathrm{ppm}\right)$. Chemical shifts are reported in parts per million as follows: chemical shift, multiplicity $(\mathrm{s}=$ singlet, $\mathrm{d}=$ doublet, $\mathrm{t}$ $=$ triplet, $\mathrm{q}=$ quartet, $\mathrm{p}=$ pentet, sext $=$ sextet, $\mathrm{m}=$ multiplet, $b r=$ broad, and combinations thereof), coupling constant and integration. Integrals are in accordance with assignments; coupling constants are given in Hz. For detailed peak assignments, 2D spectra were recorded when necessary (COSY, DEPT, HMQC, HMBC, TOCSY, GOESY). IR spectra were measured on a JASCO FT/IR-4100 instrument equipped with an ATR unit. High-resolution ESI analyses were performed on a Varian Inc. Ionspec QFT-7. Optical rotation measurements were performed on a P-2000 polarimeter from Jasco in a $10 \mathrm{~cm}$ optical-path length cell with the frequency of the $\mathrm{Na} D$ line measured at the temperature and concentration (in $\mathrm{g} / 100 \mathrm{~mL}$ ) indicated. Melting points were measured with a Stuart melting point apparatus SMP30 and are uncorrected. 
2. Scheme SI1: Proposed Mechanism of the intramolecular Minisci Reaction $\mathrm{L}_{n} \mathrm{Fe}(\mathrm{III}) \underset{-\mathrm{PhSiH}_{2}(\mathrm{O} i-\mathrm{Pr})}{\stackrel{\mathrm{PhSiH}_{3}, i-\mathrm{PrOH}}{\longrightarrow}} \quad \mathrm{L}_{n} \mathrm{Fe}(\mathrm{III})-\mathrm{H}$<smiles>C=C1CC[C@H]2C(C)(C)CCCC2(C)[C@H]1CCc1cccnc1Cl</smiles>

10
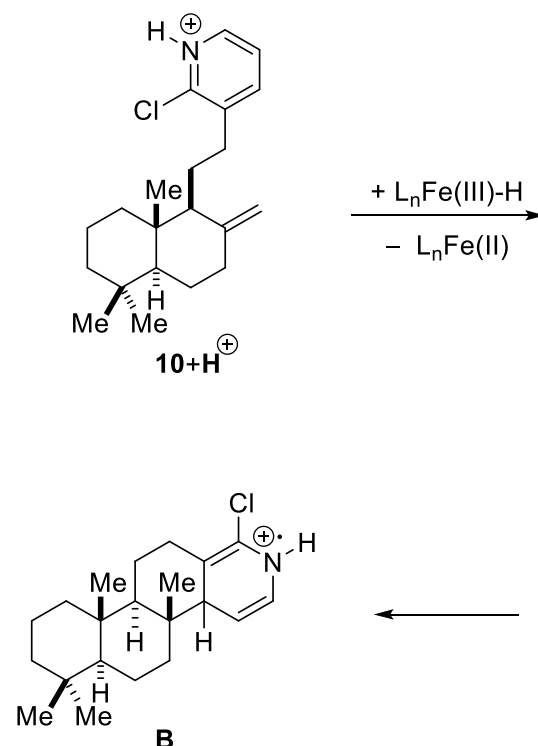

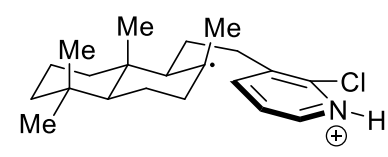

A

$+\mathrm{L}_{\mathrm{n}} \mathrm{Fe}(\mathrm{III}) \downarrow-\mathrm{L}_{\mathrm{n}} \mathrm{Fe}(\mathrm{II})$

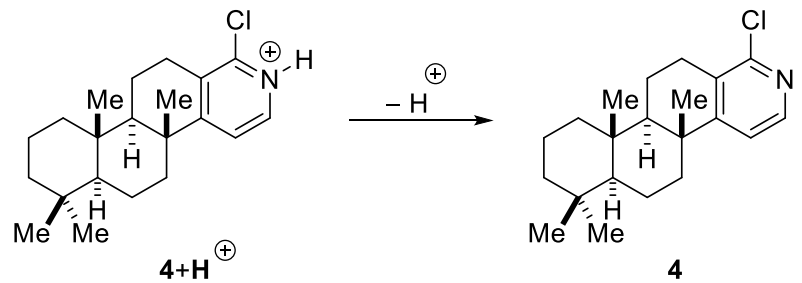




\section{Table SI1: Optimization Study for the Synthesis of Chloropyridine 4}

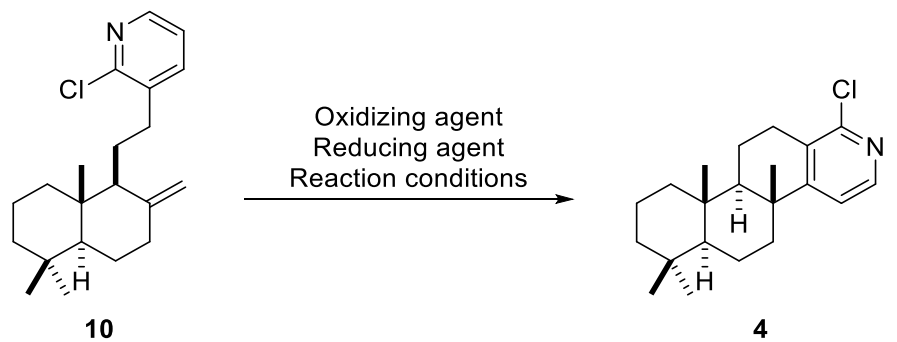

\begin{tabular}{|c|c|c|c|c|}
\hline Entry & $\begin{array}{l}\text { Oxidizing } \\
\text { agent }\end{array}$ & Reducing agent & Reaction conditions & $\begin{array}{c}\text { Observation/ } \\
\text { Yield }\end{array}$ \\
\hline 1 & $\begin{array}{l}\mathrm{Fe}(\mathrm{acac})_{3} \\
(50 \mathrm{~mol} \%)\end{array}$ & $\mathrm{PhSiH}_{3}$ (2.5 eq.) & $\begin{array}{c}(t-\mathrm{BuO})_{2}(3.0 \text { eq. }), \text { TFA (2.0 } \\
\text { eq. }), \mathrm{EtOH}, 60^{\circ} \mathrm{C}, 16 \mathrm{~h}\end{array}$ & Decomposition \\
\hline 2 & $\begin{array}{c}\mathrm{Fe}(\mathrm{acac})_{3} \\
(2.0 \text { eq. })\end{array}$ & $\mathrm{PhSiH}_{3}$ (2.2 eq.) & $\begin{array}{c}\text { TFA (2.0 eq.), EtOH, } 60{ }^{\circ} \mathrm{C}, \\
16 \mathrm{~h}\end{array}$ & Decomposition \\
\hline 3 & $\begin{array}{l}\mathrm{Fe}(\mathrm{acac})_{3} \\
(1.0 \text { eq. })\end{array}$ & $\mathrm{PhSiH}_{3}$ (2.5 eq.) & $\begin{array}{l}(t \text {-BuO })_{2}(3.0 \text { eq. }), \text { TFA }(2.0 \\
\text { eq. }), 2 \text {-Propanol, } 23^{\circ} \mathrm{C}, 16 \mathrm{~h}\end{array}$ & Decomposition \\
\hline 4 & $\begin{array}{l}\mathrm{Fe}(\mathrm{acac})_{3} \\
(1.0 \text { eq. })\end{array}$ & $\mathrm{PhSiH}_{3}$ (2.5 eq.) & $\begin{array}{l}\text { TFA (1.0 eq.), 2-Propanol, } \\
223^{\circ} \mathrm{C}, 16 \mathrm{~h}\end{array}$ & Decomposition \\
\hline 5 & $\begin{array}{c}\mathrm{Fe}(\mathrm{acac})_{3} \\
(1.0 \text { eq. })\end{array}$ & $\mathrm{PhSiH}_{3}$ (1.0 eq.) & $\begin{array}{c}\mathrm{BF}_{3} \cdot \mathrm{Et}_{2} \mathrm{O}(2.0 \text { eq. }), \\
\mathrm{THF} / \mathrm{MeOH}(4: 1), 60{ }^{\circ} \mathrm{C}, \\
16 \mathrm{~h}\end{array}$ & Decomposition \\
\hline 6 & $\begin{array}{c}\mathrm{Fe}(\mathrm{acac})_{3} \\
\text { (3.0 eq.) }\end{array}$ & $\mathrm{PhSiH}_{3}$ (3.0 eq.) & $\begin{array}{c}\mathrm{BF}_{3} \cdot \mathrm{Et}_{2} \mathrm{O}(2.0 \text { eq. }), \\
\mathrm{THF} / \mathrm{MeOH}(5: 1), 150{ }^{\circ} \mathrm{C}, \\
30 \mathrm{~min}\end{array}$ & Decomposition \\
\hline 7 & $\begin{array}{c}2 \times \mathrm{Fe}(\mathrm{dibm})_{3} \\
\quad(1.0 \text { eq. })\end{array}$ & $\begin{array}{l}2 \times \mathrm{PhSiH}_{3} \\
\text { (2.5 eq.) }\end{array}$ & $\begin{array}{l}\text { TFA (1.0 eq.), 2-Propanol, } \\
120^{\circ} \mathrm{C}, 15 \mathrm{~min} \text {, then TFA } \\
(1.0 \text { Eq. }), 120^{\circ} \mathrm{C}, 15 \mathrm{~min}\end{array}$ & $37 \%$ \\
\hline 8 & $\begin{array}{l}2 \times \mathrm{Fe}(\mathrm{acac})_{3} \\
\quad(1.0 \text { eq. })\end{array}$ & $\begin{array}{c}\mathrm{PhSiH}_{2}(\mathrm{O} i-\mathrm{Pr}) \\
(2.5 \text { eq. }+20 \text { eq. })\end{array}$ & $\begin{array}{l}\text { TFA (1.0 eq.), 2-Propanol, } \\
120^{\circ} \mathrm{C}, 20 \mathrm{~min} \text {, then TFA } \\
(1.0 \text { eq. }), 120^{\circ} \mathrm{C}, 4.5 \mathrm{~h}\end{array}$ & $19 \%$ \\
\hline 9 & $\begin{array}{l}2 \times \mathrm{Fe}(\mathrm{acac})_{3} \\
\quad(1.0 \text { eq. })\end{array}$ & $\begin{array}{l}2 \times \mathrm{PhSiH}_{3} \\
\text { (2.5 eq.) }\end{array}$ & $\begin{array}{l}\text { TFA (1.3 eq.), 2-Propanol, } \\
120^{\circ} \mathrm{C}, 10 \mathrm{~min} \text {, then TFA } \\
(1.3 \text { eq. }), 120^{\circ} \mathrm{C}, 20 \mathrm{~min}\end{array}$ & $49 \%$ \\
\hline
\end{tabular}

Note 1.: Initial cyclization attempts utilizing the $N$-phthalimidoyl oxalate of alcohol 5 \{A: $\left[\mathrm{Ru}(\text { bpy })_{3} \mathrm{Cl}_{2}\right]$, Hantzester, DIPEA, TFA, $\mathrm{CH}_{2} \mathrm{Cl}_{2}, 23^{\circ} \mathrm{C}$, blue LED; B: [ $\left.\operatorname{Ir}(\text { ppy })_{3}\right]$, TFA, $\mathrm{CH}_{2} \mathrm{Cl}_{2}, 23{ }^{\circ} \mathrm{C}$, blue LED] were unproductive and led to the recovery of 5 by cleavage of the oxalate moiety. 
Note 2.: The use of $\mathrm{Co}(\mathrm{acac})_{2}$ instead of $\mathrm{Fe}(\mathrm{acac})_{3}$ was unsuccessful under the optimized reaction conditions. 


\section{Synthesis of Compounds}

\section{Ether $\mathbf{S 1}$}

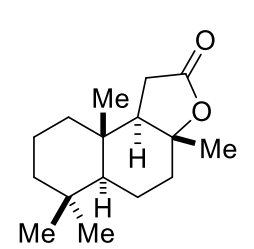

(+)-sclareolide

$\mathrm{C}_{16} \mathrm{H}_{26} \mathrm{O}_{2}$

(250.38)
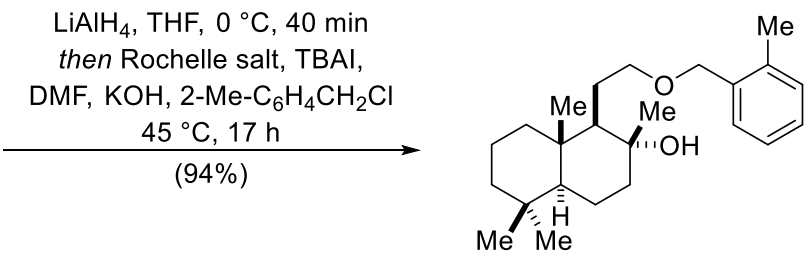

s1

$\mathrm{C}_{24} \mathrm{H}_{38} \mathrm{O}_{2}$

(358.56)

To $\mathrm{LiAlH}_{4}\left(535 \mathrm{mg}, 14.1 \mathrm{mmol}, 0.70\right.$ eq.) in THF $(5 \mathrm{~mL})$ at $0{ }^{\circ} \mathrm{C}$ was added a solution of (+)-sclareolide (5.04 g, $20.1 \mathrm{mmol}, 1.0$ eq.) in THF (25 mL) over $5 \mathrm{~min}$. After $30 \mathrm{~min}$ at the same temperature Rochelle salt $(6.84 \mathrm{~g}, 24.2 \mathrm{mmol}, 1.2 \mathrm{eq}),. \quad \mathrm{DMF} \quad(80 \mathrm{~mL})$, tetrabutylammonium iodide $(751 \mathrm{mg}, 2.03 \mathrm{mmol}, 10 \mathrm{~mol} \%)$, freshly ground $\mathrm{KOH}(4.51 \mathrm{~g}$, $80.5 \mathrm{mmol}$, 4.0 eq.) and 2- $\mathrm{Me}-\mathrm{C}_{6} \mathrm{H}_{4} \mathrm{CH}_{2} \mathrm{Cl}(10.5 \mathrm{~mL}, 60.4 \mathrm{mmol}, 3.0$ eq. $)$ were added successively, and the reaction mixture was heated to $45^{\circ} \mathrm{C}$ for $17 \mathrm{~h}$. Water was added, and the mixture was diluted with EtOAc. The organic phase was separated, and the aqueous phase was extracted with EtOAc. The combined organic extracts were washed repeatedly with brine, dried over $\mathrm{MgSO}_{4}$, filtered and concentrated under reduced pressure. The residue was purified by flash column chromatography $\left(\mathrm{SiO}_{2}, n\right.$-pentane/EtOAc 10:1) to afford ether $\mathbf{S 1}(6.78 \mathrm{~g}$, $18.9 \mathrm{mmol}, 94 \%$ ) as a colorless oil.

${ }^{1} \mathbf{H}-\mathbf{N M R}\left(500 \mathrm{MHz}, \mathrm{CDCl}_{3}\right): \delta[\mathrm{ppm}]=7.30-7.28(\mathrm{~m}, 1 \mathrm{H}), 7.22-7.14(\mathrm{~m}, 3 \mathrm{H}), 4.58-4.48$ $(\mathrm{m}, 2 \mathrm{H}), 3.64(\mathrm{ddd}, J=8.7,5.4,4.1 \mathrm{~Hz}, 1 \mathrm{H}), 3.38$ (ddd, $J=10.1,8.7,4.5 \mathrm{~Hz}, 1 \mathrm{H}), 3.24(\mathrm{sbr}$, $1 \mathrm{H}), 2.34(\mathrm{~s}, 3 \mathrm{H}), 1.90(\mathrm{dt}, J=12.5,3.2 \mathrm{~Hz}, 1 \mathrm{H}), 1.77(\mathrm{ddt}, J=15.3,10.2,5.2 \mathrm{~Hz}, 1 \mathrm{H}), 1.68-$ $1.53(\mathrm{~m}, 4 \mathrm{H}), 1.46-1.34(\mathrm{~m}, 3 \mathrm{H}), 1.30-1.20(\mathrm{~m}, 2 \mathrm{H}), 1.18-1.10(\mathrm{~m}, 4 \mathrm{H}), 0.93-0.82(\mathrm{~m}$, $5 \mathrm{H}), 0.79(\mathrm{~s}, 6 \mathrm{H})$.

The spectral data matched the previously obtained data. ${ }^{1}$ 
Alkene 6
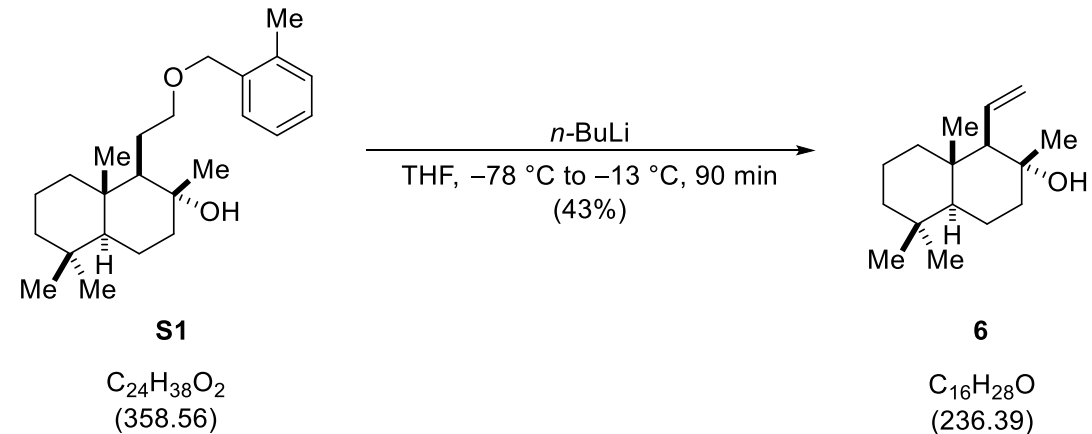

To a solution of ether $\mathbf{S 1}$ (7.67 g, $21.4 \mathrm{mmol}, 1.0$ eq.) in THF $(170 \mathrm{~mL})$ at $-78{ }^{\circ} \mathrm{C}$ was added a solution of $n$-BuLi $(34.2 \mathrm{~mL}, 2.5 \mathrm{M}$ in hexane, $85.6 \mathrm{mmol}, 4.0$ eq.). After $10 \mathrm{~min}$ at that temperature, the reaction mixture was warmed to $-13{ }^{\circ} \mathrm{C}$ (change of cooling baths) and stirred for $90 \mathrm{~min}$. Water was added, and the reaction mixture was diluted with EtOAc. The organic phase was separated, and the aqueous phase was extracted with EtOAc. The combined organic extracts were washed with brine, dried over $\mathrm{MgSO}_{4}$, filtered and concentrated under reduced pressure. The residue was purified by flash column chromatography $\left(\mathrm{SiO}_{2}, n\right.$-pentane/EtOAc 1:0 to $15: 1$ to $10: 1)$ to afford alkene $6(2.19 \mathrm{~g}, 9.24 \mathrm{mmol}, 43 \%)$ as a white solid.

${ }^{1} \mathbf{H}-\mathbf{N M R}\left(500 \mathrm{MHz}, \mathrm{CDCl}_{3}\right): \delta[\mathrm{ppm}]=5.82(\mathrm{dt}, J=16.8,10.2 \mathrm{~Hz}, 1 \mathrm{H}), 5.26(\mathrm{dd}, J=10.2$, $2.5 \mathrm{~Hz}, 1 \mathrm{H}), 5.15(\mathrm{dd}, J=16.9,2.4 \mathrm{~Hz}, 1 \mathrm{H}), 1.94(\mathrm{~s} b r, 1 \mathrm{H}), 1.90(\mathrm{dt}, J=12.6,3.3 \mathrm{~Hz}, 1 \mathrm{H}), 1.75$ $(\mathrm{d}, J=10.2 \mathrm{~Hz}, 1 \mathrm{H}), 1.71-1.64(\mathrm{~m}, 1 \mathrm{H}), 1.56(\mathrm{tt}, J=14.4,3.7 \mathrm{~Hz}, 1 \mathrm{H}), 1.51-1.36(\mathrm{~m}, 4 \mathrm{H})$, $1.38-1.23(\mathrm{~m}, 1 \mathrm{H}), 1.19(\mathrm{~s}, 3 \mathrm{H}), 1.14(\mathrm{td}, J=13.5,12.8,4.3 \mathrm{~Hz}, 1 \mathrm{H}), 0.93-0.89(\mathrm{~m}, 4 \mathrm{H})$, $0.89-0.85(\mathrm{~m}, 4 \mathrm{H}), 0.81(\mathrm{~s}, 3 \mathrm{H})$.

The spectral data matched the previously obtained data. ${ }^{1}$ 
Iodide 7
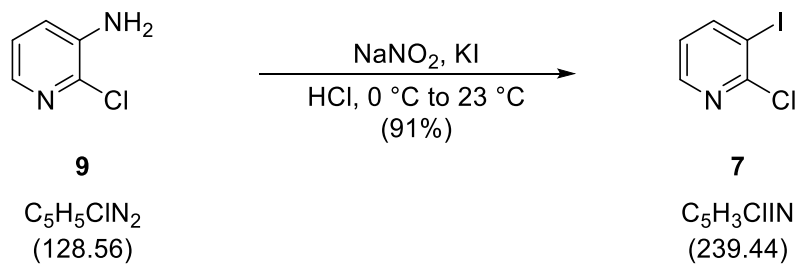

3-Amino-2-chloropyridine 9 (5.00 g, $38.9 \mathrm{mmol}, 1.0$ eq.) was added to $\mathrm{HCl}$ (6 M, aq., $40.2 \mathrm{~mL}$ ) at $0{ }^{\circ} \mathrm{C} . \mathrm{NaNO}_{2}(4.29 \mathrm{~g}, 62.2 \mathrm{mmol}, 1.6$ eq. $)$ in $\mathrm{H}_{2} \mathrm{O}(20.0 \mathrm{~mL})$ was added dropwise to the reaction mixture over $5 \mathrm{~min}$ followed by the dropwise addition of $\mathrm{KI}(15.5 \mathrm{~g}, 93.3 \mathrm{mmol}$, 2.4 eq.) in $\mathrm{H}_{2} \mathrm{O}(20.0 \mathrm{~mL})$. The reaction mixture was stirred for $5 \mathrm{~min}$ at $0{ }^{\circ} \mathrm{C}$ and $50 \mathrm{~min}$ at $23{ }^{\circ} \mathrm{C}$. $\mathrm{NaOH}(5 \mathrm{M}$, aq., $77.8 \mathrm{~mL})$ and EtOAc were added. The phases were separated, and the aqueous phase was extracted with EtOAc $(2 \mathrm{x})$. The combined organic extracts were washed consecutively with a saturated aqueous solution of $\mathrm{Na}_{2} \mathrm{~S}_{2} \mathrm{O}_{3}$ and brine. The organic phase was dried over $\mathrm{MgSO}_{4}$, filtered, and concentrated under reduced pressure. The crude product was purified by flash column chromatography $\left(\mathrm{SiO}_{2}, n\right.$-pentane/EtOAc 15:1 to 10:1) to afford the title compound $(8.33 \mathrm{~g}, 35.2 \mathrm{mmol}, 91 \%)$ as a white solid.

$\mathbf{R}_{f}=0.64$ (n-pentane/EtOAc, 5:1).

${ }^{1} \mathbf{H}-\mathbf{N M R}\left(700 \mathrm{MHz}, \mathrm{CDCl}_{3}\right): \delta[\mathrm{ppm}]=8.35(\mathrm{dd}, J=4.7,1.8 \mathrm{~Hz}, 1 \mathrm{H}), 8.13(\mathrm{dd}, J=7.8$, $1.8 \mathrm{~Hz}, 1 \mathrm{H}), 6.94(\mathrm{dd}, J=7.8,4.7 \mathrm{~Hz}, 1 \mathrm{H})$.

${ }^{13} \mathrm{C}-\mathrm{NMR}\left(176 \mathrm{MHz}, \mathrm{CDCl}_{3}\right): \delta[\mathrm{ppm}]=154.6,149.0,148.9,123.3,95.0$.

IR $\left(v / \mathrm{cm}^{-1}\right.$, ATR $)=3044,2954,2919,2851,1733,1550,1462,1382,1136,1006$.

HRMS (ESI): $m / z$ calculated for $\mathrm{C}_{5} \mathrm{H}_{4} \mathrm{ClIN}^{+}[\mathrm{M}+\mathrm{H}]^{+}:$239.9072, found 239.9069 .

Mp: $93-94{ }^{\circ} \mathrm{C}$.

The spectral data matched the previously obtained data., ${ }^{2,3}$ 

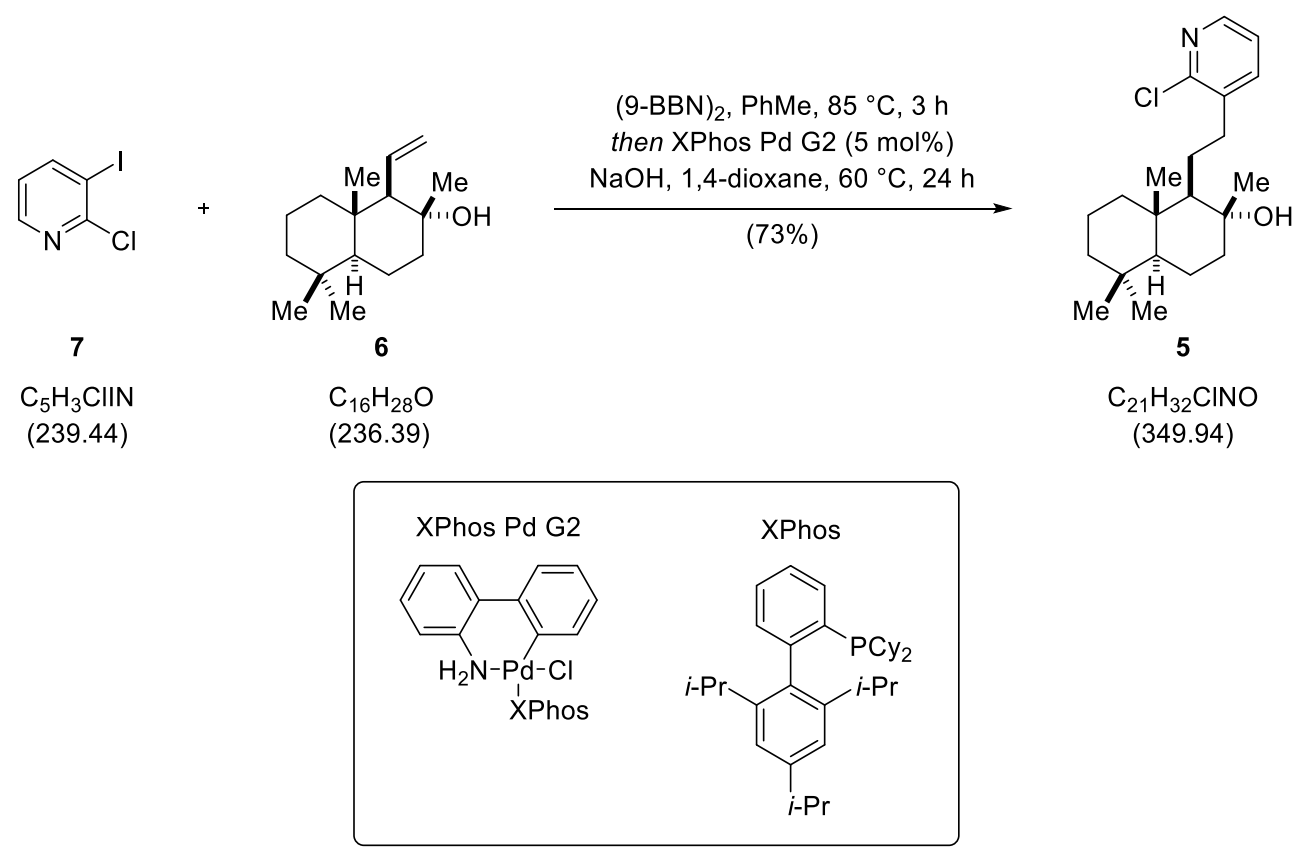

Alkene 6 (1.30 g, $5.50 \mathrm{mmol}, 1.6$ eq.) and 9-BBN dimer (1.68 g, $13.8 \mathrm{mmol}, 3.9$ eq.) were mixed and heated to $60{ }^{\circ} \mathrm{C}$ for $20 \mathrm{~min}$. The reaction mixture was heated to $85^{\circ} \mathrm{C}$ for $70 \mathrm{~min}$. Subsequently, toluene (degassed by sparging with Ar for $20 \mathrm{~min}, 3.0 \mathrm{~mL}$ ) was added, and heating was continued at $85{ }^{\circ} \mathrm{C}$ for $70 \mathrm{~min}$. After cooling to $60{ }^{\circ} \mathrm{C} \mathrm{NaOH}$ (degassed by sparging with Ar for $20 \mathrm{~min}, 7.10 \mathrm{~mL}, 3 \mathrm{M}, 21.3 \mathrm{mmol}, 6.0 \mathrm{eq}$.) was added, and the mixture was stirred for $10 \mathrm{~min}$. Afterward, 1,4-dioxane (degassed by sparging with $\mathrm{Ar}$ for $20 \mathrm{~min}, 15.0 \mathrm{ml}$ ), iodide 7 (850 mg, $3.55 \mathrm{mmol}, 1.0$ eq.) and XPhos Pd G2 (140 mg, $177 \mu \mathrm{mol}, 5 \mathrm{~mol} \%$ ) were added sequentially. The flask was rinsed with 1,4-dioxane (degassed by sparging with Ar for $20 \mathrm{~min}, 3.0 \mathrm{ml}$ ) and stirred at that temperature for $24 \mathrm{~h}$. The reaction mixture was cooled to $23{ }^{\circ} \mathrm{C}$ and water and EtOAc were added. The organic phase was separated, and the aqueous phase was extracted with EtOAc. The combined organic extracts were washed with brine, dried over $\mathrm{MgSO}_{4}$, filtered and concentrated under reduced pressure. The residue was purified by flash column chromatography $\left(\mathrm{SiO}_{2}, n\right.$-pentane/EtOAc 4:1 to 1:1) to afford the title compound (902 $\mathrm{mg}, 2.58 \mathrm{mmol}, 73 \%$ ) as a white solid.

$\mathbf{R}_{f}=0.28$ (n-pentane/EtOAc, 3:1).

$[\alpha]_{\mathrm{D}}^{27}=+31.9\left(c=0.48, \mathrm{CHCl}_{3}\right)$.

${ }^{1} \mathbf{H}-\mathbf{N M R}\left(500 \mathrm{MHz}, \mathrm{CDCl}_{3}\right): \delta[\mathrm{ppm}]=8.19(\mathrm{dd}, J=4.7,1.9 \mathrm{~Hz}, 1 \mathrm{H}), 7.57(\mathrm{dd}, J=7.5$, $1.9 \mathrm{~Hz}, 1 \mathrm{H}), 7.13(\mathrm{dd}, J=7.5,4.7 \mathrm{~Hz}, 1 \mathrm{H}), 2.85(\mathrm{ddd}, J=13.6,11.6,5.5 \mathrm{~Hz}, 1 \mathrm{H}), 2.75$ (ddd, $J$ $=13.6,11.6,5.5 \mathrm{~Hz}, 1 \mathrm{H}), 1.86(\mathrm{dt}, J=12.3,3.2 \mathrm{~Hz}, 1 \mathrm{H}), 1.78-1.73(\mathrm{~m}, 1 \mathrm{H}), 1.70-1.51(\mathrm{~m}$, $4 \mathrm{H}), 1.45-1.34(\mathrm{~m}, 4 \mathrm{H}), 1.29-1.23(\mathrm{~m}, 1 \mathrm{H}), 1.21-1.08(\mathrm{~m}, 5 \mathrm{H}), 1.00-0.91(\mathrm{~m}, 2 \mathrm{H}), 0.85$ $(\mathrm{s}, 3 \mathrm{H}), 0.76(\mathrm{~s}, 6 \mathrm{H})$. 
${ }^{13}$ C-NMR $\left(126 \mathrm{MHz}, \mathrm{CDCl}_{3}\right): \delta[\mathrm{ppm}]=.151 .2,147.1,139.0,137.4,122.7,74.4,61.6,56.2$, $44.7,42.0,39.7,39.2,36.6,33.5,33.3,25.7,24.2,21.6,20.6,18.6,15.5$.

IR $\left(v / \mathrm{cm}^{-1}, \mathrm{ATR}\right)=3411,2926,2866,2235,1565,1455,1407,1387,1079,800$.

HRMS (ESI): $m / z$ calculated for $\mathrm{C}_{21} \mathrm{H}_{33} \mathrm{ClNO}^{+}[\mathrm{M}+\mathrm{H}]^{+}: 350.2245$, found 350.2256.

Mp: $112-114{ }^{\circ} \mathrm{C}$. 
Alkene 10
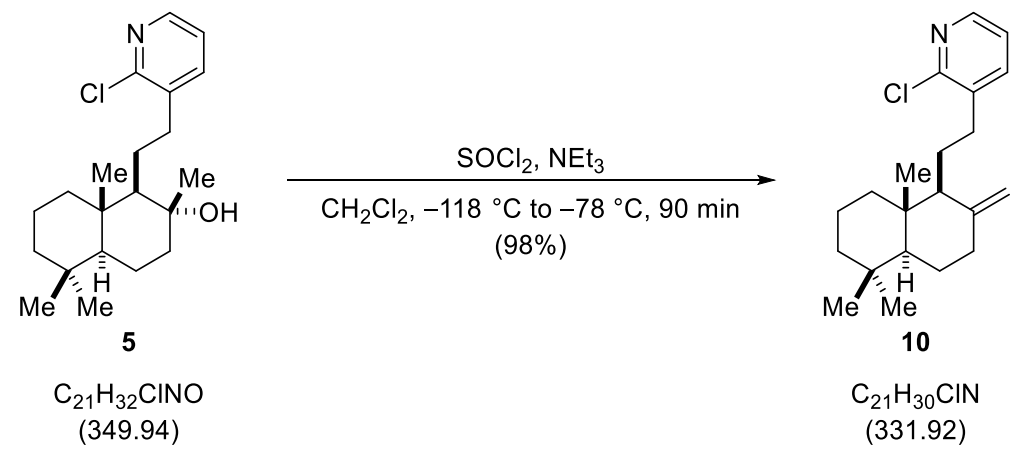

A solution of alcohol 5 (168 mg, $480 \mu \mathrm{mol}, 1.0$ eq.) and $\mathrm{NEt}_{3}$ (dried by distillation over $\mathrm{CaH}_{2}$, $0.67 \mathrm{~mL}, 0.49 \mathrm{~g}, 4.80 \mathrm{mmol}, 10.0$ eq.) in $\mathrm{CH}_{2} \mathrm{Cl}_{2}\left(16.0 \mathrm{~mL}\right.$ ) was cooled to $-118{ }^{\circ} \mathrm{C}$ (ethanol, $\mathrm{N}_{2}(\mathrm{l})$ ) and $\mathrm{SOCl}_{2}\left(0.10 \mathrm{~mL}, 0.17 \mathrm{~g}, 1.44 \mathrm{mmol}, 3.0\right.$ eq.) in $\mathrm{CH}_{2} \mathrm{Cl}_{2}$ (4.0 mL) was added dropwise over the wall of the flask over $10 \mathrm{~min}$. The reaction mixture was allowed to warm to $-78^{\circ} \mathrm{C}$ (ethanol, dry ice) over $90 \mathrm{~min}$. Methanol $(0.5 \mathrm{~mL})$ was added, and the reaction mixture was warmed to $23{ }^{\circ} \mathrm{C}$. A saturated aqueous solution of $\mathrm{NaHCO}_{3}$ and $\mathrm{CH}_{2} \mathrm{Cl}_{2}$ was added successively. The phases were separated, and the aqueous phase was extracted with $\mathrm{CH}_{2} \mathrm{Cl}_{2}$ $(2 \mathrm{x})$. The combined organic extracts were washed with brine. The organic phase was dried over $\mathrm{MgSO}_{4}$, filtered, and concentrated under reduced pressure. The crude product was purified by flash column chromatography $\left(\mathrm{SiO}_{2}, n\right.$-pentane/EtOAc 1:0 to 40:1 to 25:1 to 10:1) to afford the title compound (157 mg, $472 \mu \mathrm{mol}, 98 \%)$ as a colorless liquid, which slowly crystallized.

$\mathbf{R}_{f}=0.61$ (n-pentane/ $\left.\mathrm{CHCl}_{3}, 2: 1\right)$.

$[\alpha]_{\mathrm{D}}^{27}=+45.0\left(c=0.42, \mathrm{CHCl}_{3}\right)$.

${ }^{1} \mathbf{H}-\mathbf{N M R}\left(700 \mathrm{MHz}, \mathrm{CDCl}_{3}\right): \delta[\mathrm{ppm}]=8.26-8.22(\mathrm{~m}, 1 \mathrm{H}), 7.52(\mathrm{dd}, J=7.4,1.7 \mathrm{~Hz}, 1 \mathrm{H})$, $7.17(\mathrm{dd}, J=7.4,4.7 \mathrm{~Hz}, 1 \mathrm{H}), 4.91(\mathrm{~s}, 1 \mathrm{H}), 4.68(\mathrm{~s}, 1 \mathrm{H}), 2.92-2.85(\mathrm{~m}, 1 \mathrm{H}), 2.56-2.49(\mathrm{~m}$, $1 \mathrm{H}), 2.45-2.41(\mathrm{~m}, 1 \mathrm{H}), 2.00(\mathrm{td}, J=13.1,5.3 \mathrm{~Hz}, 1 \mathrm{H}), 1.88-1.83(\mathrm{~m}, 1 \mathrm{H}), 1.77-1.70(\mathrm{~m}$, 2H), $1.67-1.62(\mathrm{~m}, 2 \mathrm{H}), 1.57-1.52(\mathrm{~m}, 1 \mathrm{H}), 1.50-1.45(\mathrm{~m}, 1 \mathrm{H}), 1.41-1.36(\mathrm{~m}, 1 \mathrm{H}), 1.36$ $-1.30(\mathrm{~m}, 1 \mathrm{H}), 1.16(\mathrm{td}, J=13.4,4.1 \mathrm{~Hz}, 1 \mathrm{H}), 1.08(\mathrm{dd}, J=12.6,2.7 \mathrm{~Hz}, 1 \mathrm{H}), 0.97(\mathrm{td}, J=12.9$, $4.0 \mathrm{~Hz}, 1 \mathrm{H}), 0.87(\mathrm{~s}, 3 \mathrm{H}), 0.80(\mathrm{~s}, 3 \mathrm{H}), 0.68(\mathrm{~s}, 3 \mathrm{H})$.

${ }^{13}$ C-NMR $\left(176 \mathrm{MHz}, \mathrm{CDCl}_{3}\right): \delta[\mathrm{ppm}]=151.4,148.5,147.2,139.0,137.5,122.7,106.6,56.7$, $55.7,42.3,39.9,39.1,38.5,33.8(2 \mathrm{C}), 32.5,24.6,23.7,21.9,19.5,14.6$.

IR $\left(v / \mathrm{cm}^{-1}, \mathrm{ATR}\right)=2927,2846,2364,1644,1563,1456,1408,1197,1074,890$.

HRMS (ESI): $m / z$ calculated for $\mathrm{C}_{21} \mathrm{H}_{31} \mathrm{ClN}^{+}[\mathrm{M}+\mathrm{H}]^{+}: 332.2140$, found 332.2135.

Mp: $72-74{ }^{\circ} \mathrm{C}$. 


\section{Chloropyridine 4}
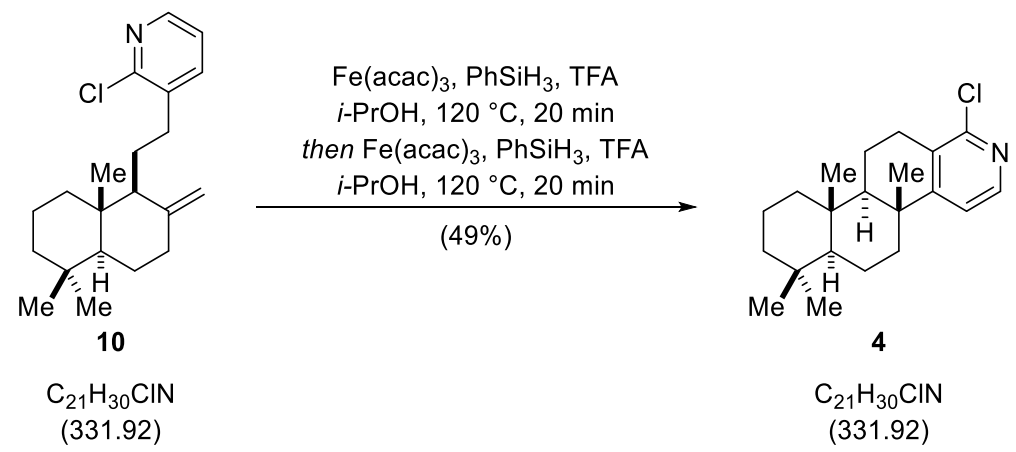

To a solution of $\mathrm{Fe}(\mathrm{acac})_{3}(15.4 \mathrm{mg}, 43.6 \mu \mathrm{mol}, 1.0$ eq.), alkene 10 (14.2 mg, $42.8 \mu \mathrm{mol}$, 1.0 eq.) and TFA ( $4.28 \mu \mathrm{L}, 6.34 \mathrm{mg}, 55.6 \mu \mathrm{mol}, 1.3 \mathrm{eq}$.) in $i$-PrOH (degassed by sparging with Ar for $20 \mathrm{~min}, 4.0 \mathrm{~mL}$ ) was added $\mathrm{PhSiH}_{3}(13.6 \mu \mathrm{L}, 11.9 \mathrm{mg}, 107 \mu \mathrm{mol}, 2.5$ eq.) and heated in a pressure tube to $120{ }^{\circ} \mathrm{C}$ for $10 \mathrm{~min}$. After cooling to $0{ }^{\circ} \mathrm{C} \mathrm{Fe}(\mathrm{acac})_{3}(15.4 \mathrm{mg}, 43.6 \mu \mathrm{mol}$, 1.0 eq.), TFA ( $4.28 \mu \mathrm{L}, 6.34 \mathrm{mg}, 55.6 \mu \mathrm{mol}, 1.3$ eq.) and $\mathrm{PhSiH}_{3}(13.6 \mu \mathrm{L}, 11.9 \mathrm{mg}, 107 \mu \mathrm{mol}$, 2.5 eq.) were added sequentially and the reaction was heated to $120{ }^{\circ} \mathrm{C}$ for $20 \mathrm{~min}$. After cooling to $23{ }^{\circ} \mathrm{C}$ the mixture was concentrated under reduced pressure, and the residue was purified by preparative thin-layer chromatography $\left(\mathrm{SiO}_{2}, n\right.$-pentane/EtOAc 70:1 and 11:1) to afford the title compound $(7.0 \mathrm{mg}, 21.1 \mu \mathrm{mol}, 49 \%)$ as a colorless solid. Crystals suitable for X-ray crystallography were obtained after the evaporation of a $\mathrm{MeCN} / \mathrm{CH}_{2} \mathrm{Cl}_{2}$ mixture.

$\mathbf{R}_{f}=0.18$ (toluene/ $\mathrm{CHCl}_{3}, 1: 1$ ).

$[\alpha]_{\mathrm{D}}^{27}=-26.7\left(c=0.48, \mathrm{CHCl}_{3}\right)$.

${ }^{1} \mathbf{H}-\mathbf{N M R}\left(500 \mathrm{MHz}, \mathrm{CDCl}_{3}\right): \delta[\mathrm{ppm}]=8.11(\mathrm{~d}, J=5.2 \mathrm{~Hz}, 1 \mathrm{H}), 7.09(\mathrm{~d}, J=5.4 \mathrm{~Hz}, 1 \mathrm{H}), 3.01$ $-2.89(\mathrm{~m}, 1 \mathrm{H}), 2.66(\mathrm{ddd}, J=18.6,11.5,7.8 \mathrm{~Hz}, 1 \mathrm{H}), 2.37-2.27(\mathrm{~m}, 1 \mathrm{H}), 1.91(\mathrm{~s}, 1 \mathrm{H}), 1.84$ $-1.78(\mathrm{~m}, 1 \mathrm{H}), 1.75-1.70(\mathrm{~m}, 1 \mathrm{H}), 1.68-1.60(\mathrm{~m}, 2 \mathrm{H}), 1.59-1.50(\mathrm{~m}, 1 \mathrm{H}), 1.48-1.43(\mathrm{~m}$, $2 \mathrm{H}), 1.42-1.38(\mathrm{~m}, 1 \mathrm{H}), 1.22-1.19(\mathrm{~m}, 1 \mathrm{H}), 1.18(\mathrm{~s}, 3 \mathrm{H}), 1.16-1.08(\mathrm{~m}, 1 \mathrm{H}), 0.92(\mathrm{~s}, 3 \mathrm{H})$, $0.88-0.86(\mathrm{~m}, 4 \mathrm{H}), 0.86-0.84(\mathrm{~m}, 4 \mathrm{H})$.

${ }^{13}$ C-NMR $\left(126 \mathrm{MHz}, \mathrm{CDCl}_{3}\right): \delta[\mathrm{ppm}]=162.3,152.0,146.5,129.9,119.1,56.2,53.9,42.1$, $40.1,39.8,38.6,37.8,33.4(2 \mathrm{C}), 28.6,25.7,21.5,19.0,18.6,17.3,16.4$.

IR $\left(v / \mathrm{cm}^{-1}, \mathrm{ATR}\right)=2925,2852,1726,1578,1542,1460,1386,1324,1105,835$.

HRMS (ESI): $m / z$ calculated for $\mathrm{C}_{21} \mathrm{H}_{31} \mathrm{ClN}^{+}[\mathrm{M}+\mathrm{H}]^{+}:$332.2140, found 332.2151.

Mp: $153-156{ }^{\circ} \mathrm{C}$. 
Pyridine 11
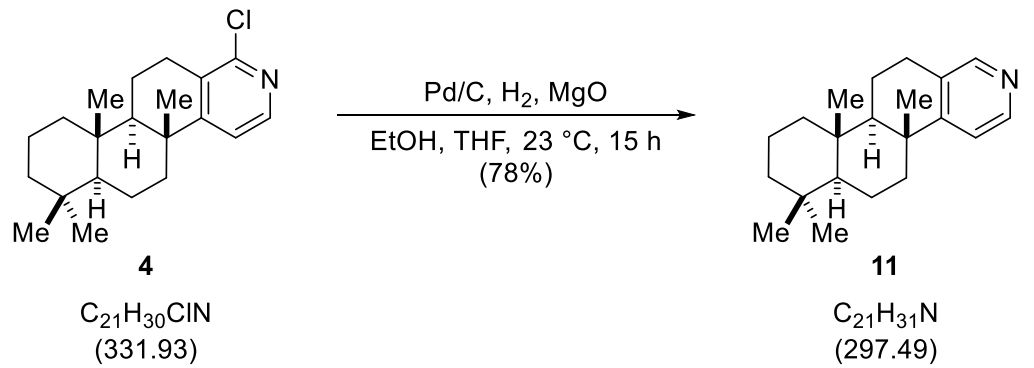

A suspension of $\mathrm{Pd} / \mathrm{C}$ (10 wt\%, $12.1 \mathrm{mg}, 11.3 \mu \mathrm{mol}, 0.4 \mathrm{eq}$.) and $\mathrm{MgO}$ (10.3 mg, $255 \mu \mathrm{mol}$, 9.0 eq.) in $\mathrm{EtOH}(1 \mathrm{~mL})$ was purged with $\mathrm{H}_{2}$ (balloon) for 2 min. Then a solution of chloropyridine 4 (9.4 mg, $28.3 \mu \mathrm{mol}, 1.0$ eq.) in THF/EtOH (1:1, $4 \mathrm{~mL})$ was added, and purging was continued for $2 \mathrm{~min}$. The reaction mixture was stirred at $23{ }^{\circ} \mathrm{C}$ for $15 \mathrm{~h}$ under an $\mathrm{H}_{2}$ (balloon) atmosphere. The suspension was filtered through a short silica pad with EtOAc/ $\mathrm{CHCl}_{3}$ $(1: 1)$ as the eluent. The mixture was concentrated under reduced pressure, and the residue was purified by flash column chromatography $\left(\mathrm{SiO}_{2}, n\right.$-pentane/EtOAc/NEt 3 150:45:2) to afford the title compound (6.6 mg, $22.2 \mu \mathrm{mol}, 78 \%)$ as a colorless solid.

$\mathbf{R}_{f}=0.41$ (n-pentane/EtOAc, $\left.1: 1\right)$.

$[\alpha]_{\mathrm{D}}^{23}=-62.6\left(c=0.07, \mathrm{CHCl}_{3}\right)\left[\mathrm{Lit}^{4}:[\alpha]_{\mathrm{D}}^{22}=-76.0\left(c=0.27, \mathrm{CHCl}_{3}\right)\right]$.

${ }^{1} \mathbf{H}-\mathbf{N M R}\left(700 \mathrm{MHz}, \mathrm{CDCl}_{3}\right): \delta[\mathrm{ppm}]=8.29(\mathrm{~d}, J=5.3 \mathrm{~Hz}, 1 \mathrm{H}), 8.26(\mathrm{~s}, 1 \mathrm{H}), 7.10(\mathrm{~d}, J=5.3$ Hz, 1H), 2.93 (dd, $J=17.3,6.4 \mathrm{~Hz}, 1 \mathrm{H}), 2.77$ (ddd, $J=17.8,11.6,7.4 \mathrm{~Hz}, 1 \mathrm{H}), 2.34$ (dt, $J=$ $12.5,3.2 \mathrm{~Hz}, 1 \mathrm{H}), 1.91(\mathrm{ddt}, J=11.7,7.7,2.0 \mathrm{~Hz}, 1 \mathrm{H}), 1.83-1.78(\mathrm{~m}, 1 \mathrm{H}), 1.76-1.71(\mathrm{~m}$, $1 \mathrm{H}), 1.70-1.61(\mathrm{~m}, 2 \mathrm{H}), 1.59-1.51(\mathrm{~m}, 1 \mathrm{H}), 1.50-1.42(\mathrm{~m}, 2 \mathrm{H}), 1.42-1.37(\mathrm{~m}, 1 \mathrm{H}), 1.28$ $-1.21(\mathrm{~m}, 1 \mathrm{H}), 1.18(\mathrm{~s}, 3 \mathrm{H}), 1.17-1.12(\mathrm{~m}, 1 \mathrm{H}), 0.93(\mathrm{~s}, 3 \mathrm{H}), 0.90-0.83(\mathrm{~m}, 8 \mathrm{H})$.

${ }^{13}$ C-NMR (176 MHz, $\left.\mathrm{CDCl}_{3}\right): \delta[\mathrm{ppm}]=158.8,150.5,147.1,130.8,119.4,56.4,54.7,42.2$, $39.9,39.8,38.1,37.9,33.5,33.4,27.8,25.8,21.6,19.0,18.7,17.6,16.5$.

IR $\left(v / \mathrm{cm}^{-1}\right.$, ATR $)=2952,2924,2855,2360,2034,1740,1697,1462,1079,830$.

HRMS (ESI): $m / z$ calculated for $\mathrm{C}_{21} \mathrm{H}_{32} \mathrm{~N}^{+}[\mathrm{M}+\mathrm{H}]^{+}:$: 298.2530, found 298.2530.

Mp: $104-106^{\circ} \mathrm{C}$. 

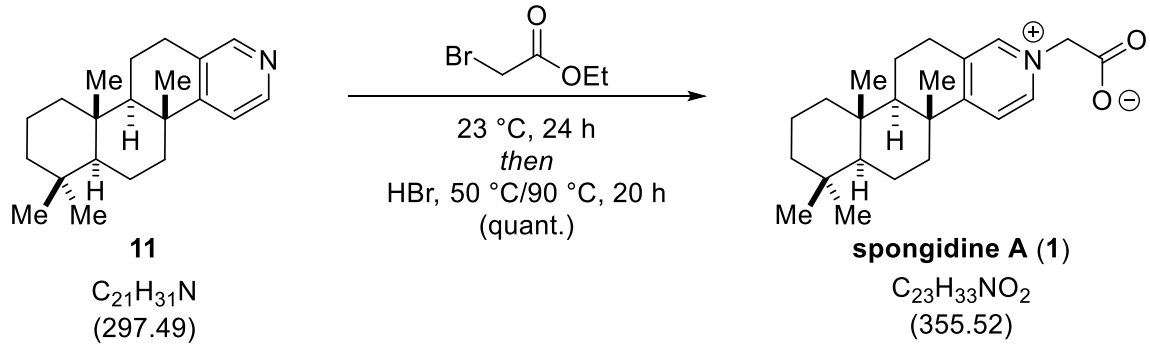

Ethyl bromoacetate $(0.359 \mathrm{~mL}, 0.539 \mathrm{~g}, 3.23 \mathrm{~mol}, 600$ eq.) was added to pyridine 11 (1.6 mg, $5.4 \mu \mathrm{mol}, 1.0$ eq.) and stirred for $24 \mathrm{~h}$ at $23{ }^{\circ} \mathrm{C}$. The reaction mixture was concentrated under reduced pressure. $\mathrm{HBr}(1.0 \mathrm{~mL})$ was added and the reaction mixture heated to $60{ }^{\circ} \mathrm{C}$ for $2 \mathrm{~h}$ in a sand bath. $\mathrm{HBr}(1.0 \mathrm{~mL})$ was added and the reaction mixture heated to $90{ }^{\circ} \mathrm{C}$ for $2 \mathrm{~h}$ and $16 \mathrm{~h}$ at $50{ }^{\circ} \mathrm{C}$ in a sand bath. The reaction mixture was concentrated under reduced pressure to afford the title compound ( $2.4 \mathrm{mg}, 6.7 \mu \mathrm{mol}$, quant.) as brown solid.

$\mathbf{R}_{f}=0.11\left(\mathrm{CH}_{2} \mathrm{Cl}_{2} / \mathrm{MeOH}, 9: 1\right)$.

$[\alpha]_{\mathrm{D}}^{25}=-32.5 \quad(c=0.21, \quad \mathrm{MeOH}) \quad\left[\mathrm{Lit}^{5}: \quad[\alpha]_{\mathrm{D}}=-16.2 \quad(c=0.010, \quad \mathrm{MeOH})\right.$, $\left.\mathrm{Lit}^{4}:[\alpha]_{\mathrm{D}}^{22}=-41.3(c=0.15, \mathrm{MeOH})\right]$.

${ }^{1} \mathbf{H}-\mathrm{NMR}\left(500 \mathrm{MHz}, \mathrm{MeOH}-d_{4}\right): \delta[\mathrm{ppm}]=8.62(\mathrm{~s}, 1 \mathrm{H}), 8.58(\mathrm{~d}, J=6.6 \mathrm{~Hz}, 1 \mathrm{H}), 7.99(\mathrm{~d}$, $J=6.6 \mathrm{~Hz}, 1 \mathrm{H}), 5.40(\mathrm{~s}, 2 \mathrm{H}), 3.16(\mathrm{dd}, J=16.8,6.8 \mathrm{~Hz}, 1 \mathrm{H}), 2.99(\mathrm{ddd}, J=18.4,11.3,7.6 \mathrm{~Hz}$, $1 \mathrm{H}), 2.53(\mathrm{dt}, J=12.5,3.3 \mathrm{~Hz}, 1 \mathrm{H}), 2.08-2.03(\mathrm{~m}, 1 \mathrm{H}), 1.89-1.66(\mathrm{~m}, 5 \mathrm{H}), 1.59(\mathrm{td}, J=12.8$, $4.0 \mathrm{~Hz}, 1 \mathrm{H}), 1.52-1.47(\mathrm{~m}, 1 \mathrm{H}), 1.45-1.41(\mathrm{~m}, 1 \mathrm{H}), 1.39-1.36(\mathrm{~m}, 1 \mathrm{H}), 1.32(\mathrm{~s}, 3 \mathrm{H}), 1.21$ (td, $J=13.6,4.2 \mathrm{~Hz}, 1 \mathrm{H}), 1.03$ (s, 3H), $0.99-0.95$ (m, 2H), 0.91 (s, 3H), 0.91 (s, 3H).

Note: $\mathrm{RCOOH}$ was not assigned.

${ }^{13}$ C-NMR $\left(176 \mathrm{MHz}, \mathrm{MeOH}-d_{4}\right): \delta[\mathrm{ppm}]=171.7,168.6,146.7,143.7,138.2,125.1,60.8$, 57.2, 54.5, 43.0, 41.0, 40.7, 40.1, 39.1, 34.3, 33.7, 28.5, 25.6, 21.8, 19.8, 19.5, 17.7, 16.9 .

IR $\left(v / \mathrm{cm}^{-1}\right.$, ATR $)=3385,2924,2852,1739,1642,1506,1458,1366,1217,1076$.

HRMS (ESI): $m / z$ calculated for $\mathrm{C}_{23} \mathrm{H}_{34} \mathrm{NO}_{2}{ }^{+}[\mathrm{M}+\mathrm{H}]^{+}:$356.2584, found 356.2586. 


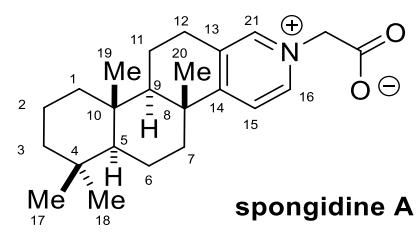

Table 2: NMR comparison of natural spongidine A with synthetic material.

\begin{tabular}{|c|c|c|c|c|}
\hline \multirow[b]{2}{*}{ No. } & \multicolumn{2}{|c|}{ natural $^{5}$} & \multicolumn{2}{|l|}{ synthetic } \\
\hline & ${ }^{1} \mathrm{H}-\mathrm{NMR}{ }^{\mathrm{a}}$ & ${ }^{13} \mathrm{C}-\mathrm{NMR}^{\mathrm{b}}$ & ${ }^{1} \mathrm{H}-\mathrm{NMR}^{\mathrm{c}}$ & ${ }^{13} \mathrm{C}-\mathrm{NMR}{ }^{\mathrm{d}}$ \\
\hline \multirow[t]{2}{*}{1} & 0.99 & 40.6 & $0.98 \mathrm{~m}$ & 40.7 \\
\hline & 1.88 & & $1.86 \mathrm{~m}^{\mathrm{e}}$ & \\
\hline \multirow[t]{2}{*}{2} & 1.54 & 19.6 & $1.51 \mathrm{~m}$ & 19.8 \\
\hline & 1.77 & & $1.75 \mathrm{~m}^{\mathrm{e}}$ & \\
\hline \multirow[t]{2}{*}{3} & 1.23 & 43.2 & $1.21 \operatorname{td}(13.6,4.2)$ & 43.0 \\
\hline & 1.45 & & $1.44 \mathrm{~m}$ & \\
\hline 4 & / & 34.3 & I & 34.3 \\
\hline 5 & 0.99 & 57.3 & $0.98 \mathrm{~m}$ & 57.2 \\
\hline \multirow[t]{2}{*}{6} & 1.70 & 19.4 & $1.70 \mathrm{~m}^{\mathrm{e}}$ & 19.5 \\
\hline & 1.83 & & $1.84 \mathrm{~m}^{\mathrm{e}}$ & \\
\hline \multirow[t]{2}{*}{7} & 1.58 & 40.4 & $1.59 \operatorname{td}(12.8,4.0)$ & 40.1 \\
\hline & $2.53 \mathrm{dd}(12.4,3.0)$ & & $2.53 \mathrm{dt}(12.5,3.3)$ & \\
\hline 8 & l & 40.5 & l & 41.0 \\
\hline 9 & 1.36 & 54.7 & $1.35 \mathrm{~m}$ & 54.5 \\
\hline 10 & l & 40.0 & l & 39.1 \\
\hline \multirow[t]{2}{*}{11} & 1.80 & 18.0 & $1.81 \mathrm{~m}$ & 17.7 \\
\hline & $2.05 \mathrm{~m}$ & & $2.05 \mathrm{~m}$ & \\
\hline \multirow[t]{2}{*}{12} & $2.97 \mathrm{~m}$ & 28.5 & 2.99 ddd $(18.4,11.3,7.6)$ & 28.5 \\
\hline & $3.15 \mathrm{dd}(18.0,6.2)$ & & $3.16 \mathrm{dd}(16.8,6.8)$ & \\
\hline 13 & I & 137.7 & I & 138.2 \\
\hline 14 & I & 170.2 & I & 171.7 \\
\hline 15 & $7.90 \mathrm{~d}(6.3)$ & 124.7 & $7.99 \mathrm{~d}(6.6)$ & 125.1 \\
\hline 16 & $8.49 \mathrm{~d}(6.3)$ & 143.3 & $8.58 \mathrm{~d}(6.6)$ & 143.7 \\
\hline 17 & $0.93 \mathrm{~s}$ & 33.8 & $0.91 \mathrm{~s}$ & 33.7 \\
\hline 18 & $0.93 \mathrm{~s}$ & 21.9 & $0.91 \mathrm{~s}$ & 21.8 \\
\hline 19 & $1.04 \mathrm{~s}$ & 16.8 & $1.03 \mathrm{~s}$ & 16.9 \\
\hline 20 & $1.32 \mathrm{~s}$ & 25.6 & $1.32 \mathrm{~s}$ & 25.6 \\
\hline 21 & $8.53 \mathrm{~s}$ & 146.3 & $8.62 \mathrm{~s}$ & 146.7 \\
\hline$-\mathrm{CH}_{2} \mathrm{COOH}$ & I & 170.5 & I & 168.6 \\
\hline$-\mathrm{CH}_{2} \mathrm{COOH}$ & $5.07 \mathrm{~s}$ & 63.9 & $5.40 \mathrm{~s}$ & 60.8 \\
\hline
\end{tabular}

All chemical shifts are reported in ppm. Coupling constants are in parentheses and are reported in $\mathrm{Hz}$. $\mathrm{m}=$ centered multiplet. All spectra are measured in $\mathrm{MeOD}$ and are referenced to the residual solvent 
peak. ${ }^{a}$ Recorded at $500 \mathrm{MHz} .{ }^{\mathrm{b}}$ Recorded at $125 \mathrm{MHz} .{ }^{\mathrm{c}}$ Recorded at $700 \mathrm{MHz} .{ }^{\mathrm{d}}$ Recorded at $176 \mathrm{MHz}$. ${ }^{\mathrm{e}}$ Signal overlapping.

Comment on the comparison between the published NMR-data of the natural sample and the synthetic material: We observed a discrepancy, especially in the pyridinium ion moiety and the acetic acid substituent. We believe the unreported counterion of the pyridinium ion might cause the observed differences. The NMR-data of the synthetic material obtained by Basabe and coworkers is in good agreement with our material (only ${ }^{13} \mathrm{C}-\mathrm{NMR}$ data available, Table 3 ). ${ }^{6}$ 
Table 3: NMR comparison of synthetic spongidine A (Basabe and coworkers) with synthetic material from this work.

\begin{tabular}{|c|c|c|}
\hline No. & $\begin{array}{c}\text { Basabe and coworkers }{ }^{6} \\
{ }^{13} \mathrm{C}-\mathrm{NMR}^{\mathrm{a}}\end{array}$ & $\begin{array}{l}\text { This work } \\
{ }^{13} \mathrm{C}^{-N}{ }^{\mathrm{b}}\end{array}$ \\
\hline 1 & 40.7 & 40.7 \\
\hline 2 & 19.8 & 19.8 \\
\hline 3 & 43.0 & 43.0 \\
\hline 4 & 34.2 & 34.3 \\
\hline 5 & 57.2 & 57.2 \\
\hline 6 & 19.5 & 19.5 \\
\hline 7 & 40.1 & 40.1 \\
\hline 8 & 41.0 & 41.0 \\
\hline 9 & 54.8 & 54.5 \\
\hline 10 & 39.1 & 39.1 \\
\hline 11 & 17.7 & 17.7 \\
\hline 12 & 28.5 & 28.5 \\
\hline 13 & 138.1 & 138.2 \\
\hline 14 & 171.6 & 171.7 \\
\hline 15 & 125.1 & 125.1 \\
\hline 16 & 143.7 & 143.7 \\
\hline 17 & 21.8 & 33.7 \\
\hline 18 & 33.7 & 21.8 \\
\hline 19 & 16.9 & 16.9 \\
\hline 20 & 25.6 & 25.6 \\
\hline 21 & 146.7 & 146.7 \\
\hline$-\mathrm{CH}_{2} \mathrm{COOH}$ & 168.7 & 168.6 \\
\hline$-\mathrm{CH}_{2} \mathrm{COOH}$ & 60.9 & 60.8 \\
\hline
\end{tabular}

All chemical shifts are reported in ppm. Coupling constants are in parentheses and are reported in $\mathrm{Hz}$. All spectra are measured in MeOD and are referenced to the residual solvent peak. ${ }^{\text {a }}$ Recorded at $100 \mathrm{MHz} .{ }^{\mathrm{b}}$ Recorded at $176 \mathrm{MHz}$. 

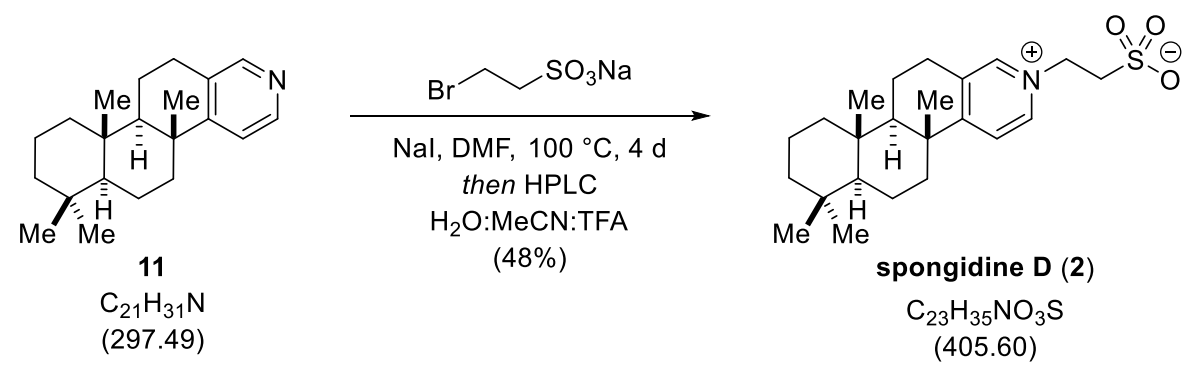

To a solution of pyridine $11(3.3 \mathrm{mg}, 11 \mu \mathrm{mol}, 1.0$ eq.) in DMF $(0.2 \mathrm{~mL})$ was added $\mathrm{NaI}$ (38 mg, $0.26 \mathrm{mmol}, 23$ eq.) and sodium 2-bromoethanesulfonate (18 mg, $83 \mu \mathrm{mol}, 7.5 \mathrm{eq}$. ) and stirred at $100{ }^{\circ} \mathrm{C}$ in a sand bath for $24 \mathrm{~h}$. Sodium 2-bromoethanesulfonate $(18 \mathrm{mg}, 83 \mu \mathrm{mol}$, 7.5 eq.) was added, and the reaction mixture was stirred at $100^{\circ} \mathrm{C}$ in a sand bath for $3 \mathrm{~d}$. The reaction mixture was concentrated under reduced pressure, and the residue was diluted with $\mathrm{MeCN}$ and filtered. The filtrate was concentrated and purified by reverse phase HPLC (MeCN: $\mathrm{H}_{2} \mathrm{O} 70: 30+0.1 \%$ TFA, flowrate: $0.7 \mathrm{~mL} / \mathrm{min}$ ) to afford the title compound $(2.3 \mathrm{mg}$, $5.4 \mu \mathrm{mol}, 48 \%$ ) as colorless solid.

$\mathbf{R}_{f}=0.36\left(\mathrm{CH}_{2} \mathrm{Cl}_{2} / \mathrm{MeOH}, 9: 1\right)$.

$[\alpha]_{\mathrm{D}}^{23}=-17.8 \quad(c=0.23, \quad \mathrm{MeOH}) \quad\left[\mathrm{Lit}^{5}: \quad[\alpha]_{\mathrm{D}}=-6 \quad(c=0.016, \quad \mathrm{MeOH})\right.$, $\left.\mathrm{Lit}^{4}:[\alpha]_{\mathrm{D}}^{22}=-10.1(c=0.02, \mathrm{MeOH})\right]$.

${ }^{1} \mathbf{H}-\mathbf{N M R}\left(500 \mathrm{MHz}, \mathrm{MeOH}-d_{4}\right): \delta[\mathrm{ppm}]=8.65(\mathrm{~s}, 1 \mathrm{H}), 8.62(\mathrm{~d}, J=6.6 \mathrm{~Hz}, 1 \mathrm{H}), 7.88(\mathrm{~d}$, $J=6.6 \mathrm{~Hz}, 1 \mathrm{H}), 4.80-4.77(\mathrm{~m}, 2 \mathrm{H}), 3.40(\mathrm{t}, J=6.2 \mathrm{~Hz}, 2 \mathrm{H}), 3.18-3.10(\mathrm{~m}, 1 \mathrm{H}), 2.96$ (ddd, $J=18.4,10.9,8.0 \mathrm{~Hz}, 1 \mathrm{H}), 2.52-2.47(\mathrm{~m}, 1 \mathrm{H}), 2.07-1.99(\mathrm{~m}, 1 \mathrm{H}), 1.89-1.63(\mathrm{~m}, 5 \mathrm{H}), 1.60$ $-1.40(\mathrm{~m}, 3 \mathrm{H}), 1.37-1.32(\mathrm{~m}, 1 \mathrm{H}), 1.29(\mathrm{~s}, 3 \mathrm{H}), 1.20(\mathrm{td}, J=13.6,4.1 \mathrm{~Hz}, 1 \mathrm{H}), 1.01(\mathrm{~s}, 3 \mathrm{H})$, $0.96(\mathrm{~d}, J=12.7 \mathrm{~Hz}, 2 \mathrm{H}), 0.91(\mathrm{~s}, 3 \mathrm{H}), 0.90(\mathrm{~s}, 3 \mathrm{H})$.

Note: $\mathrm{RSO}_{3} \mathrm{H}$ is not assigned.

${ }^{13}$ C-NMR (176 MHz, MeOH- $\left.d_{4}\right): \delta[\mathrm{ppm}]=170.9,146.0,142.8,137.9,124.9,58.0,57.2,54.5$, 51.5, 43.0, 40.8 (2C), 40.1, 39.1, 34.2, 33.7, 28.4, 25.5, 21.8, 19.8, 19.5, 17.8, 16.8.

IR $\left(v / \mathrm{cm}^{-1}\right.$, ATR $)=3357,2923,2849,1680,1641,1457,1385,1202,1122,1025,976$.

HRMS (ESI): $m / z$ calculated for $\mathrm{C}_{23} \mathrm{H}_{36} \mathrm{NO}_{3} \mathrm{~S}^{+}[\mathrm{M}+\mathrm{H}]^{+}: 406.2411$, found 406.2414. 


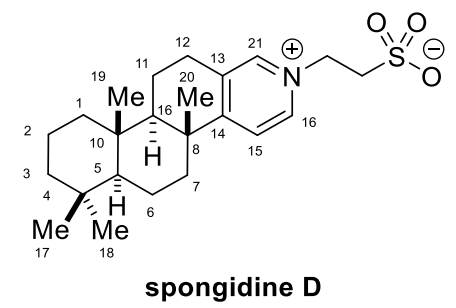

Table 4: NMR comparison of natural spongidine D with synthetic material.

\begin{tabular}{|c|c|c|c|c|}
\hline \multirow[b]{2}{*}{ No. } & \multicolumn{2}{|c|}{ natural $^{5}$} & \multicolumn{2}{|c|}{ synthetic } \\
\hline & ${ }^{1} \mathrm{H}-\mathrm{NMR}{ }^{\mathrm{a}}$ & ${ }^{13} \mathrm{C}-\mathrm{NMR}{ }^{\mathrm{b}}$ & ${ }^{1} \mathrm{H}-\mathrm{NMR}^{\mathrm{c}}$ & ${ }^{13} \mathrm{C}-\mathrm{NMR}{ }^{\mathrm{d}}$ \\
\hline \multirow[t]{2}{*}{1} & 0.97 & 40.8 & $0.96 \mathrm{~d}(12.7)$ & 40.8 \\
\hline & 1.88 & & $1.87 \mathrm{~m}^{\mathrm{e}}$ & \\
\hline \multirow[t]{2}{*}{2} & not reported & 19.9 & $1.50 \mathrm{~m}$ & 19.5 \\
\hline & not reported & & $1.85 \mathrm{~m}^{\mathrm{e}}$ & \\
\hline \multirow[t]{2}{*}{3} & 1.22 & 43.2 & $1.20 \operatorname{td}(13.6,4.1)$ & 43.0 \\
\hline & 1.46 & & $1.45 \mathrm{~m}$ & \\
\hline 4 & / & 34.6 & / & 34.2 \\
\hline 5 & 0.95 & 57.3 & $0.95 \mathrm{~d}(12.7)$ & 57.2 \\
\hline \multirow[t]{2}{*}{6} & 1.70 & 19.6 & $1.70 \mathrm{~m}^{\mathrm{e}}$ & 19.8 \\
\hline & 1.88 & & $1.85 \mathrm{~m}^{\mathrm{e}}$ & \\
\hline \multirow[t]{2}{*}{7} & 1.58 & 39.7 & $1.56 \mathrm{~m}$ & 40.1 \\
\hline & $2.50 \mathrm{dd}(12.4,3.0)$ & & $2.50 \mathrm{~m}$ & \\
\hline 8 & / & 40.2 & I & 40.8 \\
\hline 9 & 1.35 & 54.6 & $1.34 \mathrm{~m}$ & 54.5 \\
\hline 10 & I & 39.2 & I & 39.1 \\
\hline \multirow[t]{2}{*}{11} & 1.79 & 16.9 & $1.80 \mathrm{~m}$ & 17.8 \\
\hline & $2.06 \mathrm{~m}$ & & $2.03 \mathrm{~m}$ & \\
\hline \multirow[t]{3}{*}{12} & $2.98 \mathrm{~m}$ & 28.5 & 2.96 ddd (18.4, & 28.4 \\
\hline & & & $10.9,8.0)$ & \\
\hline & $3.25 \mathrm{dd}(18.0,6.2)$ & & $3.14 \mathrm{~m}$ & \\
\hline 13 & / & 137.8 & I & 137.9 \\
\hline 14 & I & 171.0 & I & 170.9 \\
\hline 15 & $7.93 \mathrm{~d}(6.3)$ & 124.9 & $7.88 \mathrm{~d}(6.6)$ & 124.9 \\
\hline 16 & $8.65 \mathrm{~d}(6.3)$ & 142.8 & $8.62 \mathrm{~d}(6.6)$ & 142.8 \\
\hline 17 & $0.93 \mathrm{~s}$ & 33.8 & $0.90 \mathrm{~s}$ & 33.7 \\
\hline 18 & $0.93 \mathrm{~s}$ & 21.3 & $0.91 \mathrm{~s}$ & 21.8 \\
\hline 19 & $1.04 \mathrm{~s}$ & 16.9 & $1.01 \mathrm{~s}$ & 16.8 \\
\hline 20 & $1.32 \mathrm{~s}$ & 25.6 & $1.29 \mathrm{~s}$ & 25.5 \\
\hline 21 & $8.68 \mathrm{~s}$ & 146.1 & $8.65 \mathrm{~s}$ & 146.0 \\
\hline$-\mathrm{CH}_{2} \mathrm{CH}_{2} \mathrm{SO}_{3} \mathrm{H}$ & $4.88 \mathrm{t}(6.2)$ & 57.5 & $4.85 \mathrm{~m}$ & 58.0 \\
\hline$-\mathrm{CH}_{2} \mathrm{CH}_{2} \mathrm{SO}_{3} \mathrm{H}$ & $3.43 \mathrm{t}(6.2)$ & 51.1 & $3.40 \mathrm{t}(6.2)$ & 51.5 \\
\hline
\end{tabular}

All chemical shifts are reported in ppm. Coupling constants are in parentheses and are reported in $\mathrm{Hz}$. $\mathrm{m}=$ centered multiplet. All spectra are measured in MeOD and are referenced to the residual solvent peak. ${ }^{\text {a }}$ Recorded at $500 \mathrm{MHz} .{ }^{\mathrm{b}}$ Recorded at $125 \mathrm{MHz} .{ }^{\mathrm{c}}$ Recorded at $700 \mathrm{MHz} .{ }^{\mathrm{d}}$ Recorded at $176 \mathrm{MHz}$. ${ }^{\mathrm{e}}$ Signal overlapping. 
Chloroiodopyridine 12

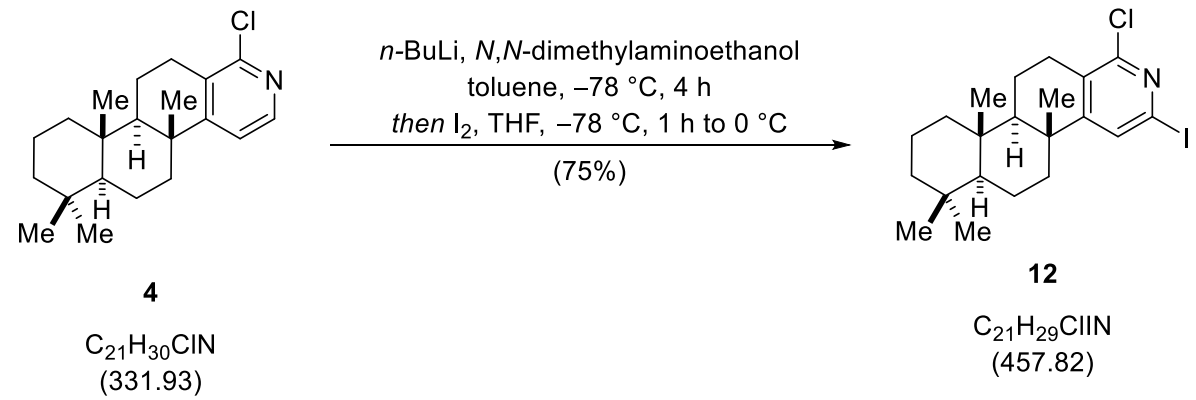

To a solution of $N, N$-dimethylaminoethanol (freshly distilled over $\mathrm{KOH}, 92.9 \mu \mathrm{L}, 82.3 \mathrm{mg}$, $923 \mu \mathrm{mol}, 8.0$ eq. $)$ in toluene $(1.0 \mathrm{~mL})$ at $-78^{\circ} \mathrm{C}$ was added $n$-BuLi $(2.5 \mathrm{M}, 739 \mu \mathrm{L}$, $1.85 \mathrm{mmol}, 16.0$ eq.) dropwise. After $15 \mathrm{~min}$, a solution of chloropyridine $4(38.3 \mathrm{mg}$, $115 \mu \mathrm{mol}, 1.0$ eq.) in toluene $(2.0 \mathrm{~mL})$ was added dropwise over $5 \mathrm{~min}$. After $4 \mathrm{~h}$ a solution of $\mathrm{I}_{2}$ (264 mg, $1.04 \mathrm{mmol}, 9.0$ eq.) in THF (1.3 mL) was added dropwise. The reaction temperature was maintained at $-78{ }^{\circ} \mathrm{C}$ for $1 \mathrm{~h}$. Then the reaction mixture was stirred for $40 \mathrm{~min}$ at $0{ }^{\circ} \mathrm{C}$ (change of cooling baths). Water $(3 \mathrm{~mL})$ and EtOAc were added, and the phases were separated, and the aqueous phase was extracted with EtOAc. The combined organic phases were washed brine, dried over $\mathrm{MgSO}_{4}$, filtered, and concentrated under reduced pressure. The residue was purified by flash column chromatography $\left(\mathrm{SiO}_{2}, n\right.$-pentane/EtOAc 1:0 to 40:1 to 25:1 to 15:1) to afford the title compound $(39.6 \mathrm{mg}, 86.5 \mu \mathrm{mol}, 75 \%)$ as a slightly yellow solid.

$\mathbf{R}_{f}=0.29$ (n-pentane/EtOAc, 50:1).

$[\alpha]_{\mathrm{D}}^{20}=-57.8\left(c=3.96, \mathrm{CHCl}_{3}\right)$.

${ }^{1} \mathbf{H}-\mathbf{N M R}\left(700 \mathrm{MHz}, \mathrm{CDCl}_{3}\right): \delta[\mathrm{ppm}]=7.49(\mathrm{~s}, 1 \mathrm{H}), 2.88(\mathrm{dd}, J=18.4,6.5 \mathrm{~Hz}, 1 \mathrm{H}), 2.58$ (ddd, $J=18.8,11.5,7.8 \mathrm{~Hz}, 1 \mathrm{H}), 2.28-2.22(\mathrm{~m}, 1 \mathrm{H}), 1.95(\mathrm{dd}, J=13.6,7.9 \mathrm{~Hz}, 1 \mathrm{H}), 1.82-$ $1.76(\mathrm{~m}, 1 \mathrm{H}), 1.75-1.71(\mathrm{~m}, 1 \mathrm{H}), 1.69-1.56(\mathrm{~m}, 2 \mathrm{H}), 1.56-1.48(\mathrm{~m}, 1 \mathrm{H}), 1.48-1.43(\mathrm{~m}$, $2 \mathrm{H}), 1.42-1.37(\mathrm{~m}, 1 \mathrm{H}), 1.18(\mathrm{~s}, 3 \mathrm{H}), 1.16-1.12(\mathrm{~m}, 2 \mathrm{H}), 0.91(\mathrm{~s}, 3 \mathrm{H}), 0.87(\mathrm{~s}, 3 \mathrm{H}), 0.85-$ $0.82(\mathrm{~m}, 5 \mathrm{H})$.

${ }^{13}$ C-NMR $\left(176 \mathrm{MHz}, \mathrm{CDCl}_{3}\right): \delta[\mathrm{ppm}]=164.0,150.9,130.4,129.8,111.4,56.1,53.6,42.0$, $40.0,39.8,38.7,37.8,33.4$ (2C), 28.2, 25.7, 21.6, 19.0, 18.6, 17.1, 16.4.

IR $\left(v / \mathrm{cm}^{-1}\right.$, ATR $)=2925,2848,2362,2094,1728,1551,1525,1458,1384,1096,1023$.

HRMS (ESI): $m / z$ calculated for $\mathrm{C}_{21} \mathrm{H}_{29} \mathrm{ClINNa}^{+}[\mathrm{M}+\mathrm{Na}]^{+}: 480.0925$, found 480.0949.

Mp: $93-95^{\circ} \mathrm{C}$. 
Alkyne 13
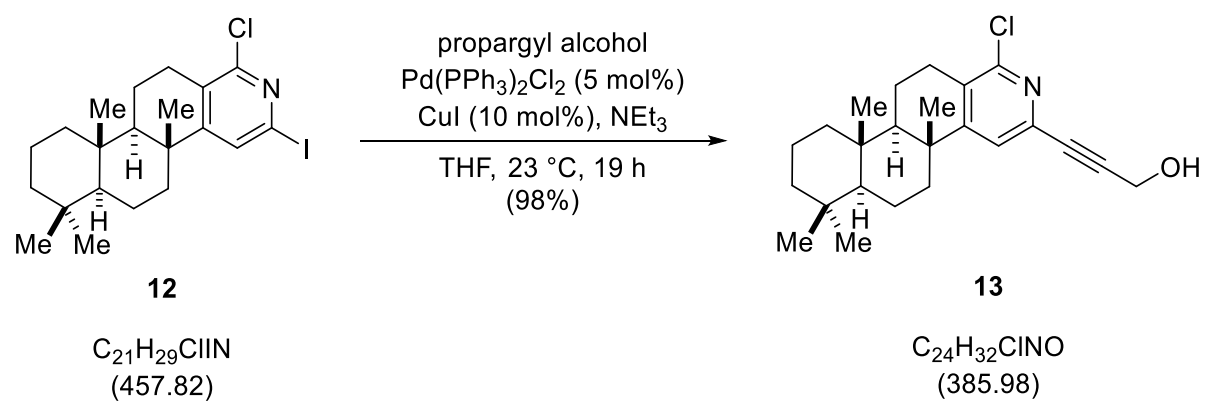

To a suspension of $\mathrm{CuI}(1.6 \mathrm{mg}, 8.37 \mu \mathrm{mol}, 10 \mathrm{~mol} \%)$ and $\mathrm{Pd}\left(\mathrm{PPh}_{3}\right) \mathrm{Cl}_{2}(2.9 \mathrm{mg}, 4.18 \mu \mathrm{mol}$, $5 \mathrm{~mol} \%)$ in $\mathrm{THF}(0.5 \mathrm{~mL})$ was added $\mathrm{NEt}_{3}(69.6 \mu \mathrm{L}, 50.8 \mathrm{mg}, 502 \mu \mathrm{mol}, 6.0$ eq. $)$ and propargyl alcohol $(14.5 \mu \mathrm{L}, 14.1 \mathrm{mg}, 251 \mu \mathrm{mol}, 3.0$ eq.) sequentially. A solution of chloroiodopyridine 12 (38.3 mg, $83.7 \mu \mathrm{mol}, 1.0$ eq.) in THF (3.0 mL) was added, and the reaction flask was stirred at $23{ }^{\circ} \mathrm{C}$ for $19 \mathrm{~h}$ in the dark. The reaction mixture was directly purified by flash column chromatography $\left(\mathrm{SiO}_{2}, n\right.$-pentane/EtOAc 10:1 to 5:1 to 2:1) to afford the title compound (31.6 mg, $81.9 \mu \mathrm{mol}, 98 \%$ ) as a slightly brown solid.

$\mathbf{R}_{f}=0.74$ (n-pentane/EtOAc, 1:1).

$[\alpha]_{\mathrm{D}}^{20}=-45.2\left(c=0.48, \mathrm{CHCl}_{3}\right)$.

${ }^{1} \mathbf{H}-\mathbf{N M R}\left(700 \mathrm{MHz}, \mathrm{CDCl}_{3}\right): \delta[\mathrm{ppm}]=7.25(\mathrm{~s}, 1 \mathrm{H}), 4.49(\mathrm{~s}, 2 \mathrm{H}), 2.94(\mathrm{dd}, J=18.6,6.2 \mathrm{~Hz}$, 1H), $2.64(\mathrm{ddd}, J=18.8,11.4,7.7 \mathrm{~Hz}, 1 \mathrm{H}), 2.28(\mathrm{~d}, J=12.1 \mathrm{~Hz}, 1 \mathrm{H}), 2.23-2.12(\mathrm{~s}$ r, $1 \mathrm{H})$, $1.95(\mathrm{dd}, J=13.5,7.5 \mathrm{~Hz}, 1 \mathrm{H}), 1.82-1.77(\mathrm{~m}, 1 \mathrm{H}), 1.75-1.69(\mathrm{~m}, 1 \mathrm{H}), 1.67-1.57(\mathrm{~m}, 2 \mathrm{H})$, $1.57-1.48(\mathrm{~m}, 1 \mathrm{H}), 1.48-1.42(\mathrm{~m}, 2 \mathrm{H}), 1.42-1.35(\mathrm{~m}, 1 \mathrm{H}), 1.16(\mathrm{~s}, 3 \mathrm{H}), 1.16-1.10(\mathrm{~m}$, $2 \mathrm{H}), 0.91(\mathrm{~s}, 3 \mathrm{H}), 0.88-0.81(\mathrm{~m}, 8 \mathrm{H})$.

${ }^{13}$ C-NMR $\left(176 \mathrm{MHz}, \mathrm{CDCl}_{3}\right): \delta[\mathrm{ppm}]=162.5,151.7,139.2,130.4,122.7,87.3,84.3,56.1$, 53.7, 51.6, 42.0, 40.0, 39.8, 38.6, 37.7, 33.4 (2C), 28.7, 25.6, 21.5, 19.0, 18.6, 17.2, 16.3.

IR $\left(v / \mathrm{cm}^{-1}\right.$, ATR $)=3377,2924,2853,1732,1577,1528,1458,1437,1379,1047$.

HRMS (ESI): $m / z$ calculated for $\mathrm{C}_{24} \mathrm{H}_{32} \mathrm{ClNONa}^{+}[\mathrm{M}+\mathrm{Na}]^{+}:$408.2064, found 408.2080.

Mp: $167-168^{\circ} \mathrm{C}$ (Decomposition). 
Alcohol 14
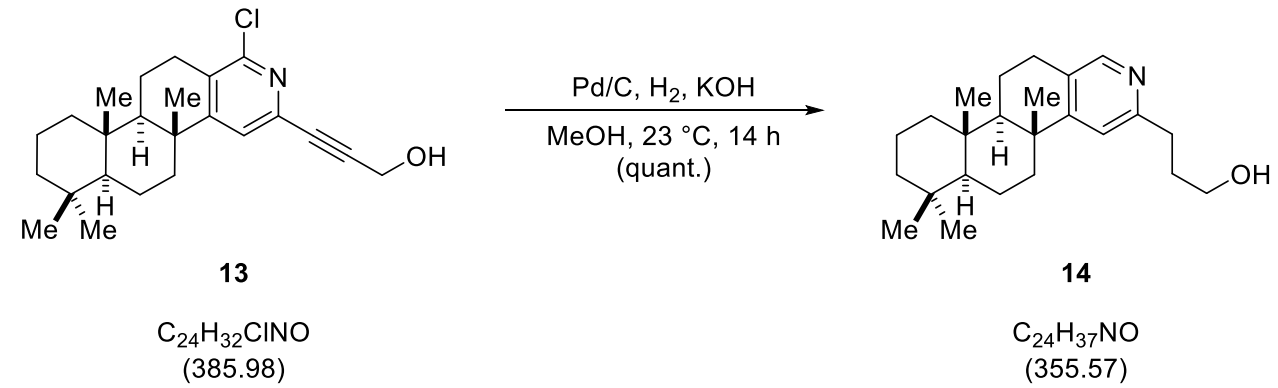

A suspension of $\mathrm{Pd} / \mathrm{C}(10 \mathrm{wt} \%, 39.7 \mathrm{mg}, 37.3 \mu \mathrm{mol}, 4.8$ eq.) and $\mathrm{KOH}(3.9 \mathrm{mg}, 70.0 \mu \mathrm{mol}$, 9.0 eq.) in $\mathrm{MeOH}\left(2 \mathrm{~mL}\right.$ ) was purged with $\mathrm{H}_{2}$ (balloon) for $2 \mathrm{~min}$. A solution of alkyne $\mathbf{1 3}$ (3.0 mg, $7.8 \mu \mathrm{mol}, 1.0$ eq.) in $\mathrm{MeOH}(4 \mathrm{~mL}$ ) was added, and purging was continued for $2 \mathrm{~min}$. The reaction mixture was stirred at $23{ }^{\circ} \mathrm{C}$ for $16 \mathrm{~h}$ under an $\mathrm{H}_{2}$ (balloon) atmosphere. The suspension was filtered through a Celite ${ }^{\circledR}$ pad with $\mathrm{CHCl}_{3}$ as the eluent. The mixture was concentrated under reduced pressure to obtain the title compound ( $3.0 \mathrm{mg}, 8.4 \mu \mathrm{mol}$, quant.) as a colorless solid.

$\mathbf{R}_{f}=0.48\left(\mathrm{CH}_{2} \mathrm{Cl}_{2} / \mathrm{MeOH}, 9: 1\right)$.

$[\alpha]_{\mathrm{D}}^{22}=-44.0\left(c=0.30, \mathrm{CHCl}_{3}\right)$.

${ }^{1} \mathbf{H}$-NMR $\left(400 \mathrm{MHz}, \mathrm{CDCl}_{3}\right): \delta[\mathrm{ppm}]=8.14(\mathrm{~s}, 1 \mathrm{H}), 6.99(\mathrm{~s}, 1 \mathrm{H}), 3.71(\mathrm{t}, J=5.7 \mathrm{~Hz}, 2 \mathrm{H})$, $2.92-2.86(\mathrm{~m}, 3 \mathrm{H}), 2.72(\mathrm{ddd}, J=17.7,11.4,7.4 \mathrm{~Hz}, 1 \mathrm{H}), 2.37-2.27$ (m, 1H), $1.99-1.85$ $(\mathrm{m}, 3 \mathrm{H}), 1.84-1.76(\mathrm{~m}, 1 \mathrm{H}), 1.76-1.68(\mathrm{~m}, 1 \mathrm{H}), 1.68-1.58(\mathrm{~m}, 2 \mathrm{H}), 1.57-1.51(\mathrm{~m}, 1 \mathrm{H})$, $1.50-1.36(\mathrm{~m}, 3 \mathrm{H}), 1.24-1.19(\mathrm{~m}, 1 \mathrm{H}), 1.17(\mathrm{~s}, 3 \mathrm{H}), 1.15-1.11(\mathrm{~m}, 2 \mathrm{H}), 0.92(\mathrm{~s}, 3 \mathrm{H}), 0.88$ $-0.81(\mathrm{~m}, 8 \mathrm{H})$.

${ }^{13}$ C-NMR $\left(176 \mathrm{MHz}, \mathrm{CDCl}_{3}\right): \delta[\mathrm{ppm}]=159.8,158.2,149.2,128.3,118.6,62.9,56.4,54.7$, $42.2,39.9$ (2C), 38.2, 37.9, 35.7, 33.5, 33.4, 31.7, 27.5, 25.8, 21.6, 19.0, 18.7, 17.6, 16.5.

IR $\left(v / \mathrm{cm}^{-1}\right.$, ATR $)=3289,2923,2855,2362,1736,1600,1547,1381,1196,1072$.

HRMS (ESI): $m / z$ calculated for $\mathrm{C}_{24} \mathrm{H}_{38} \mathrm{NO}^{+}[\mathrm{M}+\mathrm{H}]^{+}:$356.2948, found 356.2964. 
Petrosaspongiolide L methyl ester 15
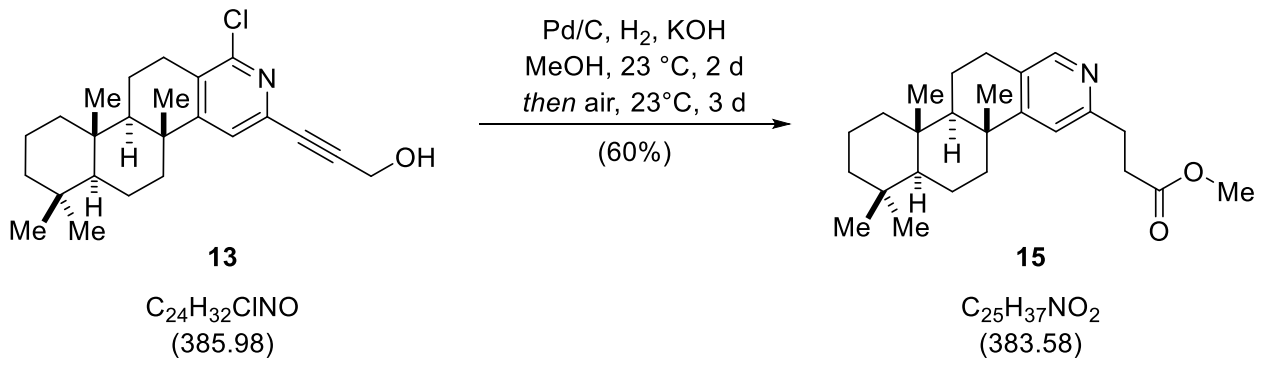

A suspension of $\mathrm{Pd} / \mathrm{C}(10 \mathrm{wt} \%, 81.6 \mathrm{mg}, 76.7 \mu \mathrm{mol}, 2.0$ eq. $)$ and $\mathrm{KOH}(10.8 \mathrm{mg}, 192 \mu \mathrm{mol}$, 5.0 eq.) in $\mathrm{MeOH}\left(3 \mathrm{~mL}\right.$ ) was purged with $\mathrm{H}_{2}$ (balloon) for $2 \mathrm{~min}$. A solution of alkyne $\mathbf{1 3}$ (14.8 mg, $38.3 \mu \mathrm{mol}, 1.0$ eq.) in $\mathrm{MeOH}(5 \mathrm{~mL})$ was added, and purging was continued for $2 \mathrm{~min}$. After $23 \mathrm{~h} \mathrm{Pd} / \mathrm{C}$ (10 wt\%, $122 \mathrm{mg}, 115 \mu \mathrm{mol}, 3.0$ eq.) and $\mathrm{KOH}$ (12.9 mg, $230 \mu \mathrm{mol}$, 6.0 eq.) were added, and the reaction mixture was purged with $\mathrm{H}_{2}$ (balloon). After stirring under an $\mathrm{H}_{2}$ atmosphere for $16 \mathrm{~h} \mathrm{Pd} / \mathrm{C}(10 \mathrm{wt} \%, 122 \mathrm{mg}, 115 \mu \mathrm{mol}, 3.0$ eq.) and $\mathrm{KOH}$ (19.4 mg, $345 \mu \mathrm{mol}, 9.0$ eq.) were added, and the reaction mixture was purged with $\mathrm{H}_{2}$ (balloon). After $16 \mathrm{~h}$ the $\mathrm{H}_{2}$ atmosphere was exchanged, and air was slowly introduced. After $3 \mathrm{~d}$ the suspension was filtered through a Celite ${ }^{\circledR}$ pad with $\mathrm{CHCl}_{3}$. The mixture was concentrated under reduced pressure and purified by preparative TLC $\left(\mathrm{SiO}_{2}, n\right.$-pentane/EtOAc 11:4) to afford the title compound (8.8 mg, $22.9 \mu \mathrm{mol}, 60 \%)$ as a colorless solid.

$\mathbf{R}_{f}=0.72($ EtOAc $)$

$[\alpha]_{\mathrm{D}}^{22}=-43.5\left(c=0.62, \mathrm{CHCl}_{3}\right)$.

${ }^{1} \mathbf{H}-\mathbf{N M R}\left(700 \mathrm{MHz}, \mathrm{CDCl}_{3}\right): \delta[\mathrm{ppm}]=8.17(\mathrm{~s}, 1 \mathrm{H}), 7.01(\mathrm{~s}, 1 \mathrm{H}), 3.66(\mathrm{~s}, 3 \mathrm{H}), 3.04(\mathrm{t}, J=7.6$ $\mathrm{Hz}, 2 \mathrm{H}), 2.89(\mathrm{dd}, J=17.0,6.3 \mathrm{~Hz}, 1 \mathrm{H}), 2.77(\mathrm{t}, J=7.6 \mathrm{~Hz}, 2 \mathrm{H}), 2.74-2.68(\mathrm{~m}, 1 \mathrm{H}), 2.38-$ $2.30(\mathrm{~m}, 1 \mathrm{H}), 1.89(\mathrm{dd}, J=13.4,7.5 \mathrm{~Hz}, 1 \mathrm{H}), 1.82-1.77(\mathrm{~m}, 1 \mathrm{H}), 1.75-1.71(\mathrm{~m}, 1 \mathrm{H}), 1.69-$ $1.59(\mathrm{~m}, 2 \mathrm{H}), 1.58-1.50(\mathrm{~m}, 1 \mathrm{H}), 1.48-1.43(\mathrm{~m}, 2 \mathrm{H}), 1.42-1.37(\mathrm{~m}, 1 \mathrm{H}), 1.23-1.18(\mathrm{~m}$, $1 \mathrm{H}), 1.17$ (s, 3H), $1.15-1.12(\mathrm{~m}, 1 \mathrm{H}), 0.93(\mathrm{~s}, 3 \mathrm{H}), 0.88-0.83(\mathrm{~m}, 8 \mathrm{H})$.

${ }^{13}$ C-NMR $\left(176 \mathrm{MHz}, \mathrm{CDCl}_{3}\right): \delta[\mathrm{ppm}]=173.8,159.8,156.7,149.6,128.7,118.6,56.4,54.7$, 51.8, 42.2, 39.9 (2C), 38.2, 37.9, 33.9, 33.5, 33.4, 32.8, 27.5, 25.8, 21.6, 19.0, 18.7, 17.6, 16.5.

IR $\left(v / \mathrm{cm}^{-1}\right.$, ATR $)=2925,2851,2363,1739,1598,1550,1441,1371,1250,911$.

HRMS (ESI): $m / z$ calculated for $\mathrm{C}_{25} \mathrm{H}_{38} \mathrm{NO}_{2}{ }^{+}[\mathrm{M}+\mathrm{H}]^{+}: 384.2897$, found 384.2900. 
Petrosaspongiolide L (3)

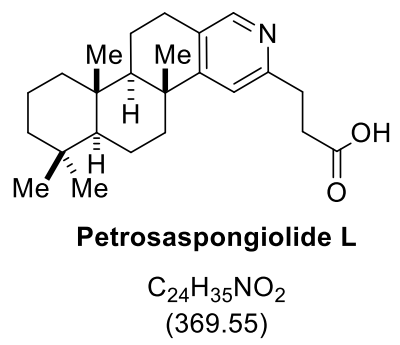

HRMS (ESI): $m / z$ calculated for $\mathrm{C}_{24} \mathrm{H}_{36} \mathrm{NO}_{2}[\mathrm{M}+\mathrm{H}]^{+}: 370.2741$, found 370.2759 . 
5. NMR-Spectra
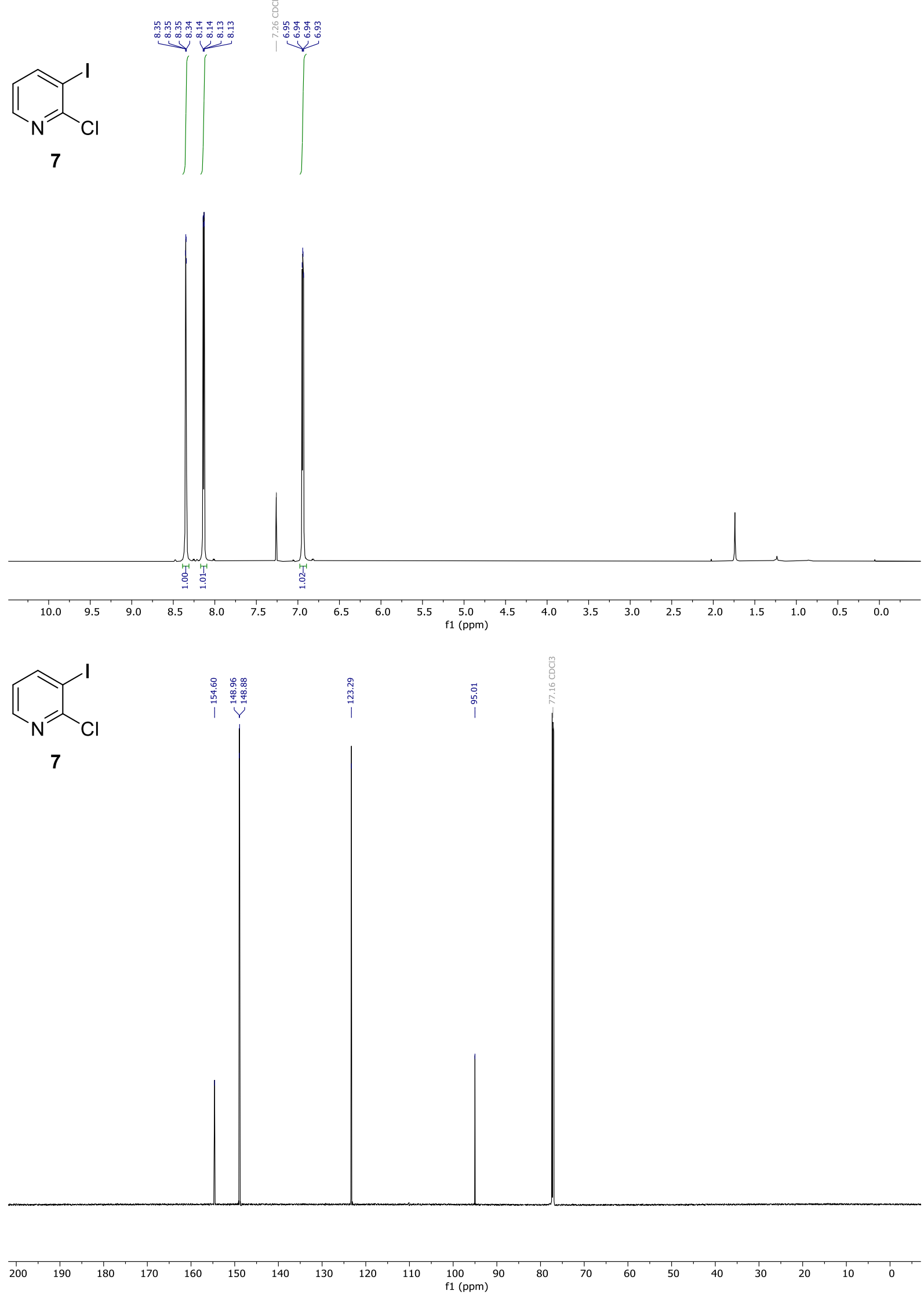

26 
<smiles>CC1(C)CCCC2(C)C1CCC(C)(O)C2CCc1cccnc1Cl</smiles>
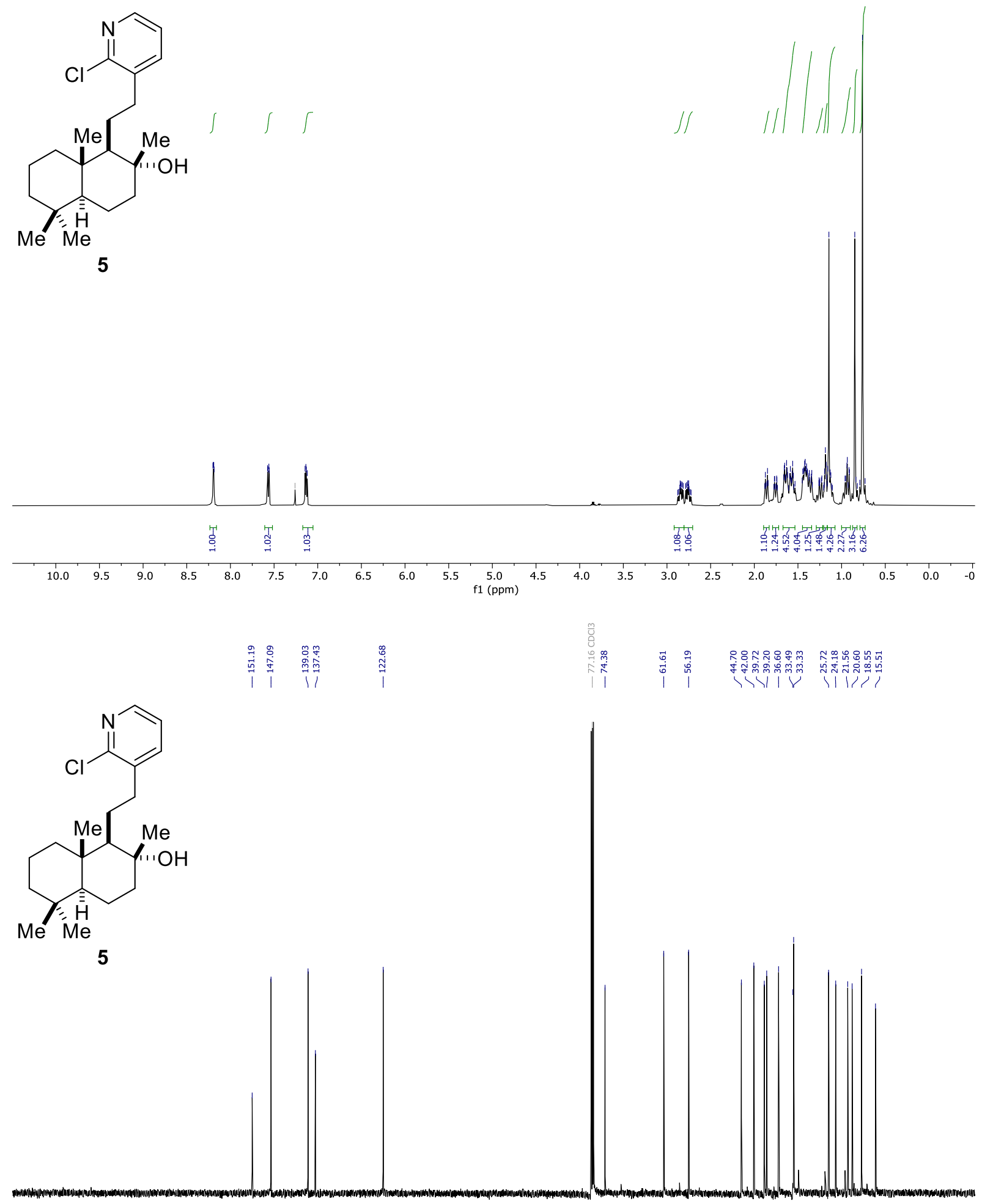

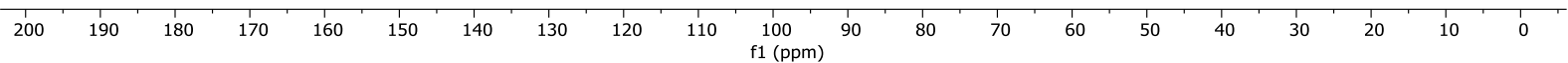


<smiles>C=C1CCC2C(C)(C)CCCC2(C)C1CCc1cccnc1Cl</smiles>
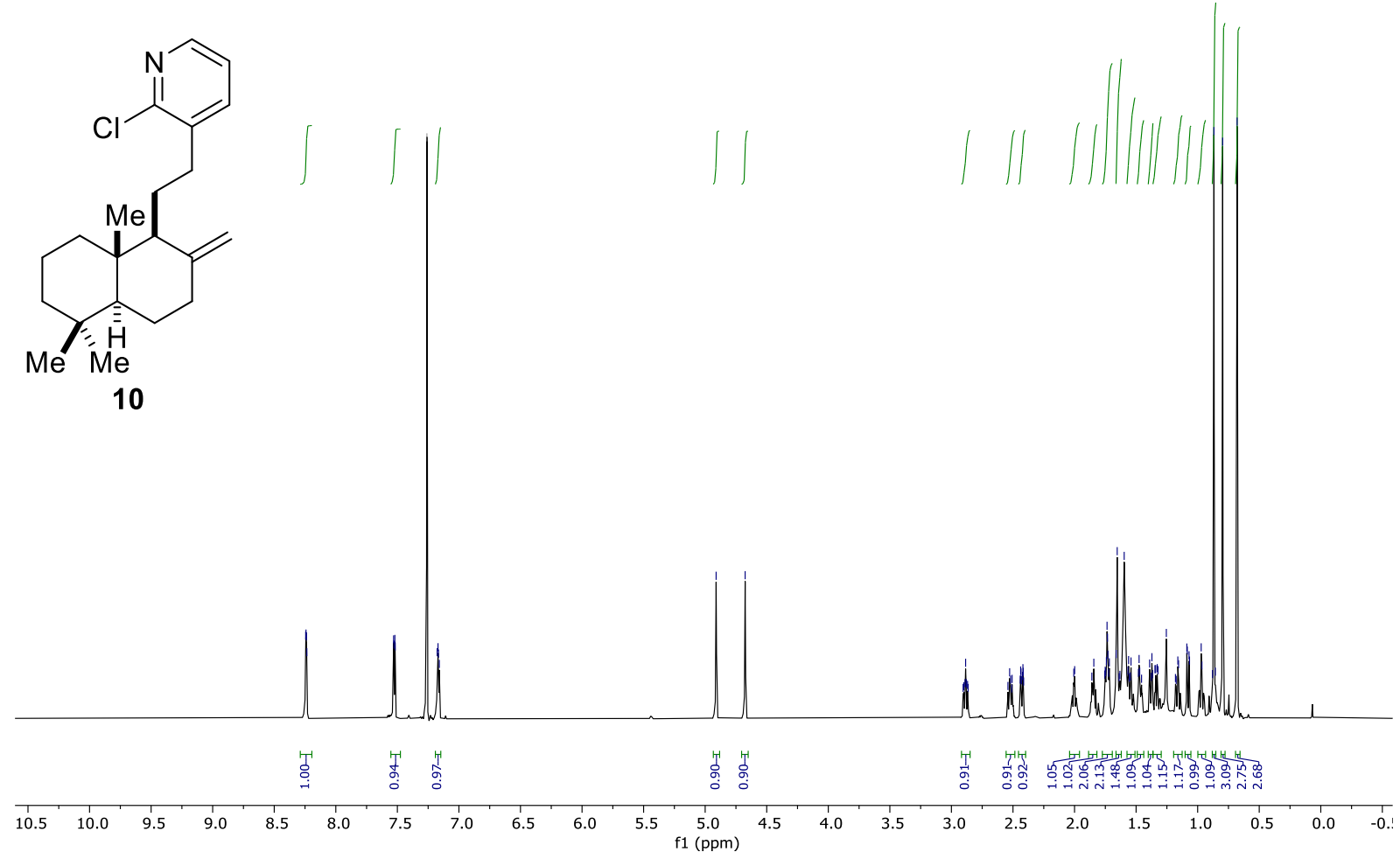<smiles>C=C1CCC2C(C)(C)CCCC2(C)C1CCc1cccnc1Cl</smiles>

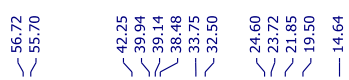

10 

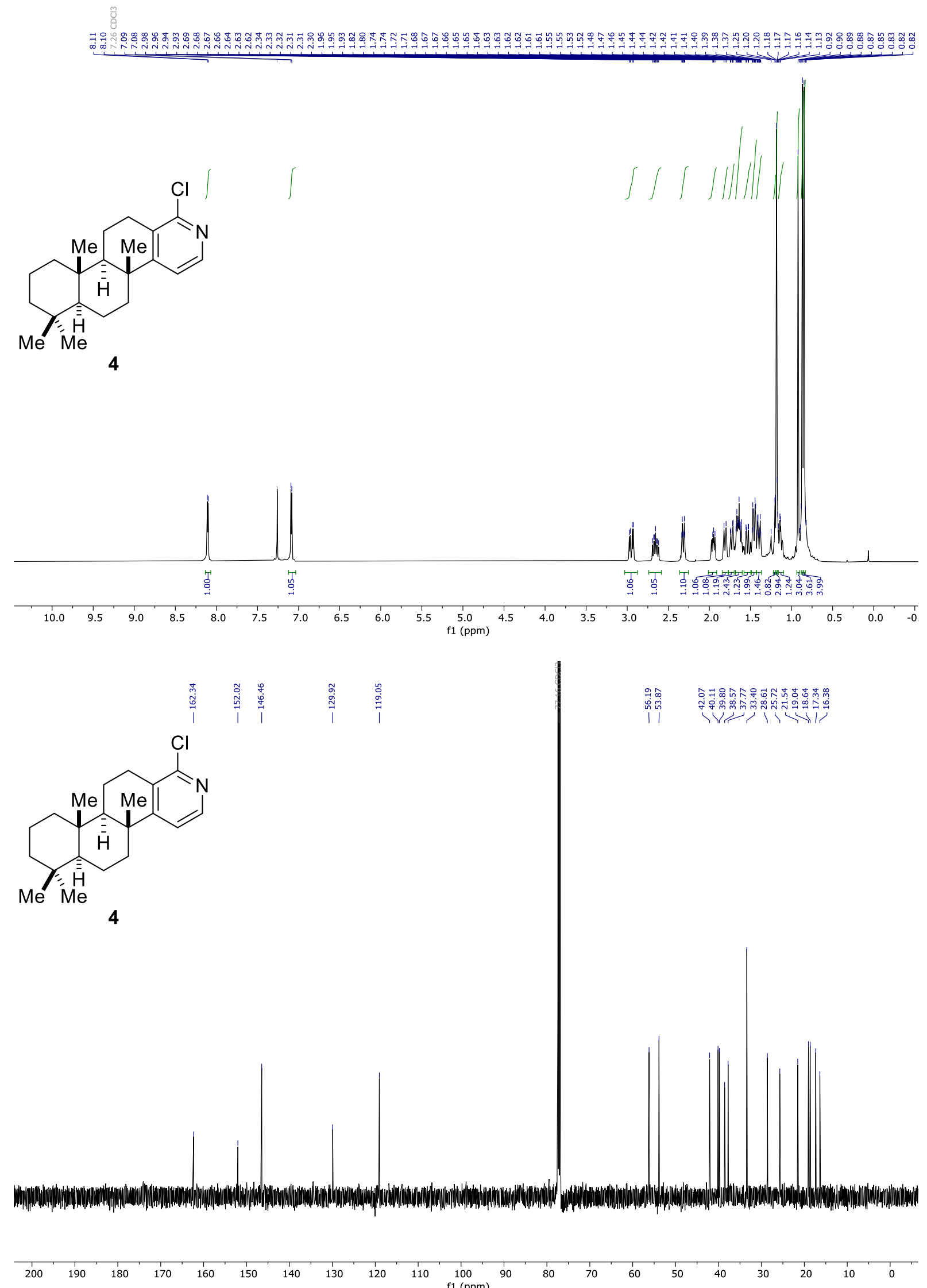
<smiles>[M]C1CCc2cnccc2C1(C)CCC1CCCCC1(C)C</smiles>

11
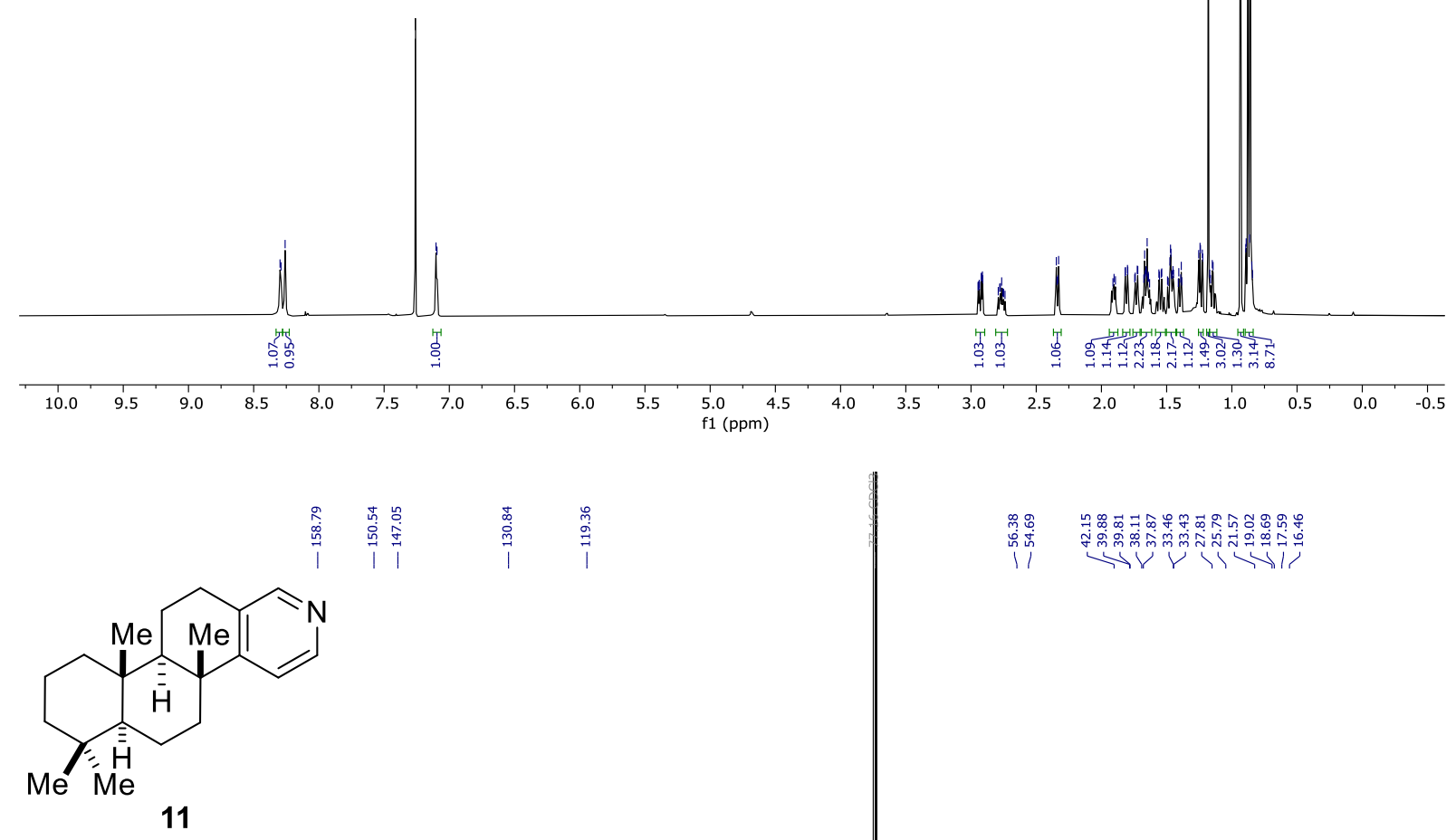

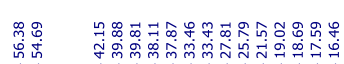

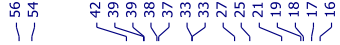

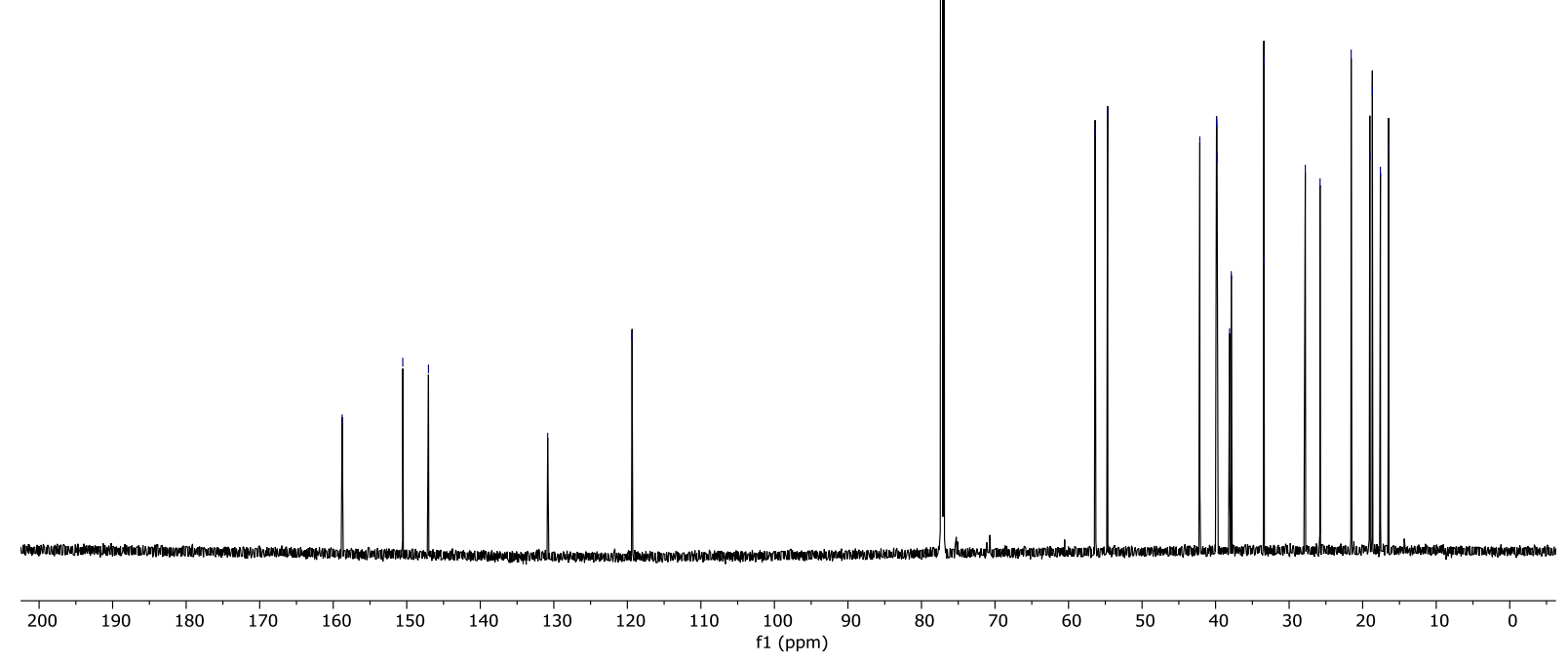



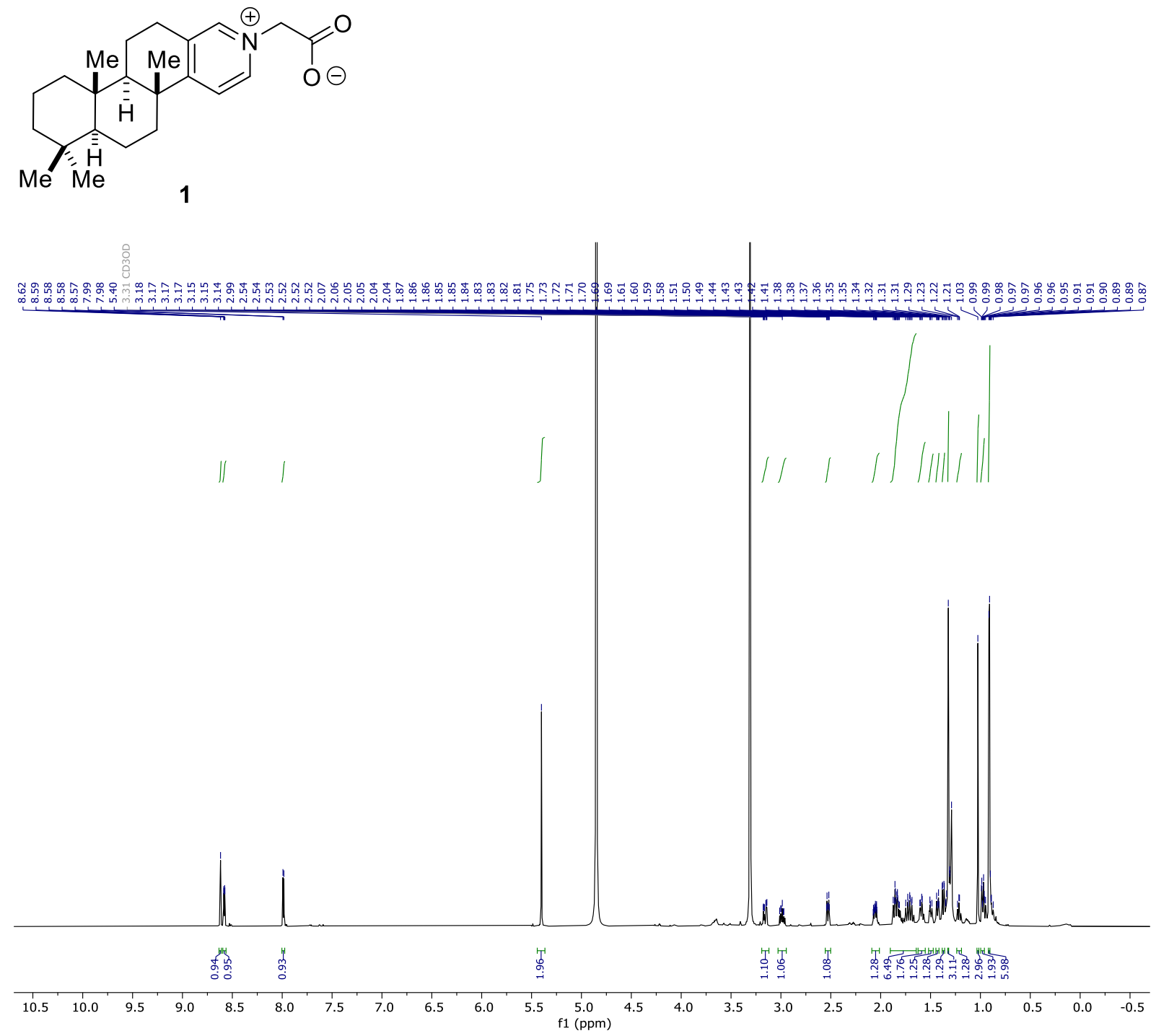

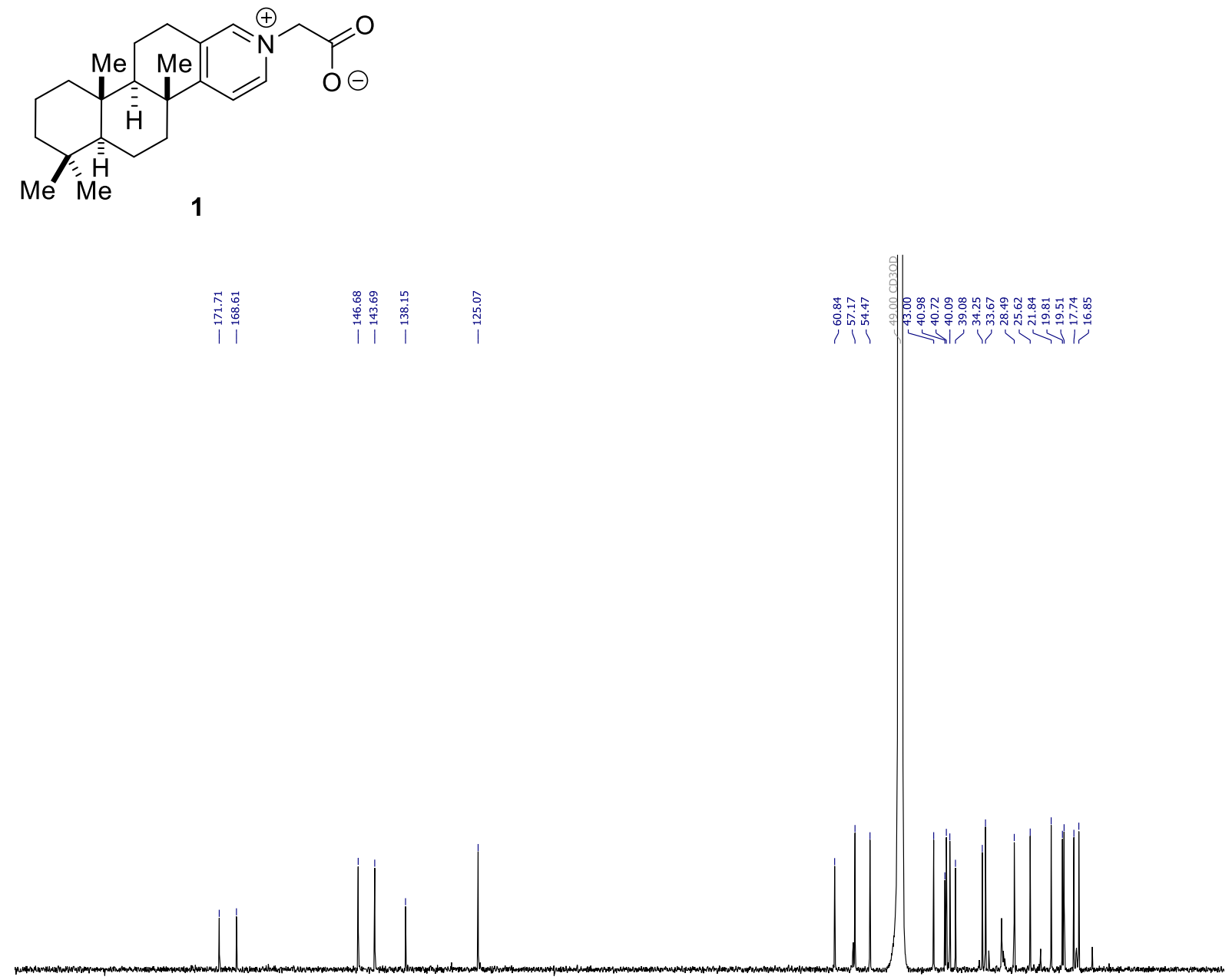
HMQC of spongidine A (1)

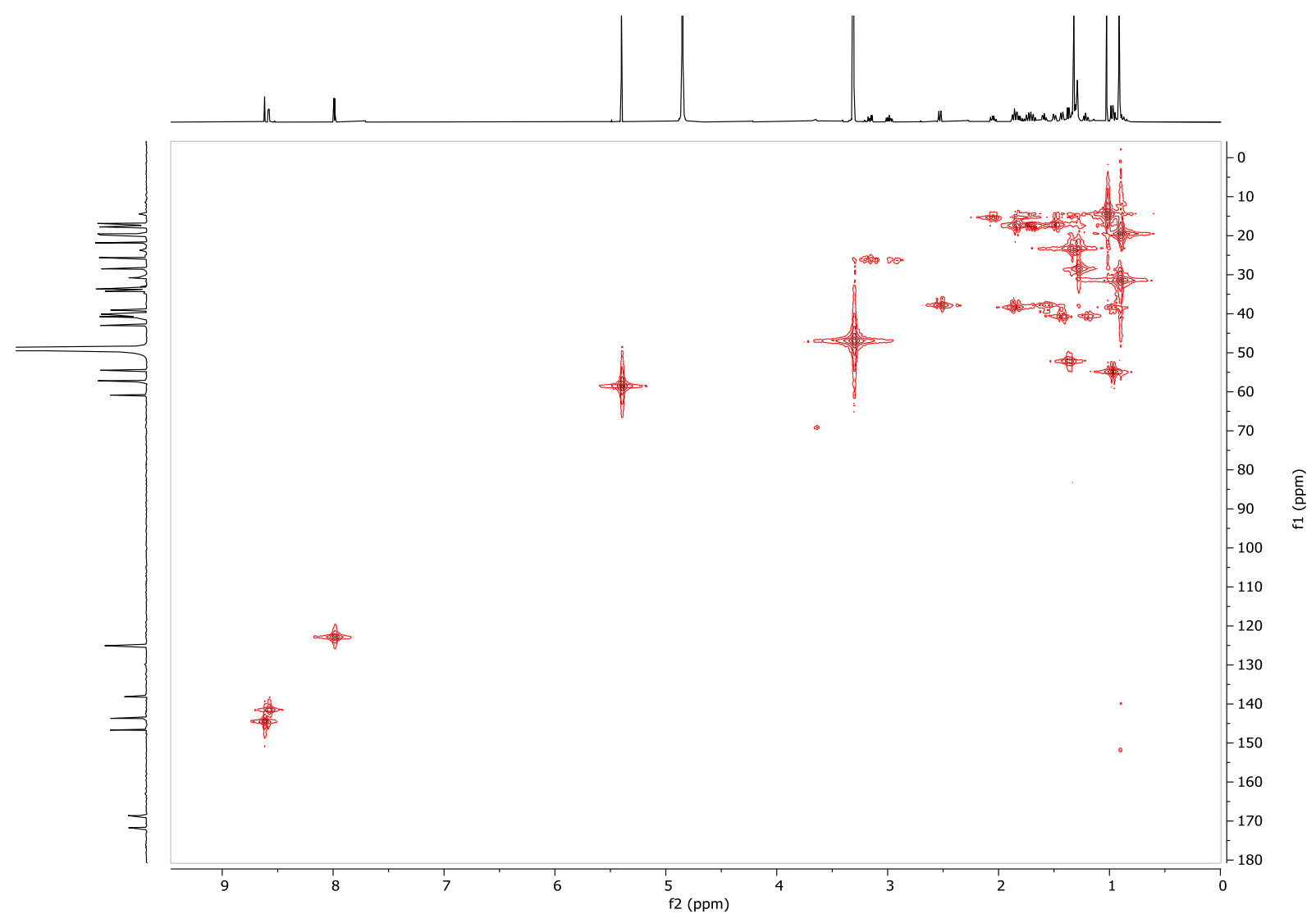


HMBC of spongidine A (1)

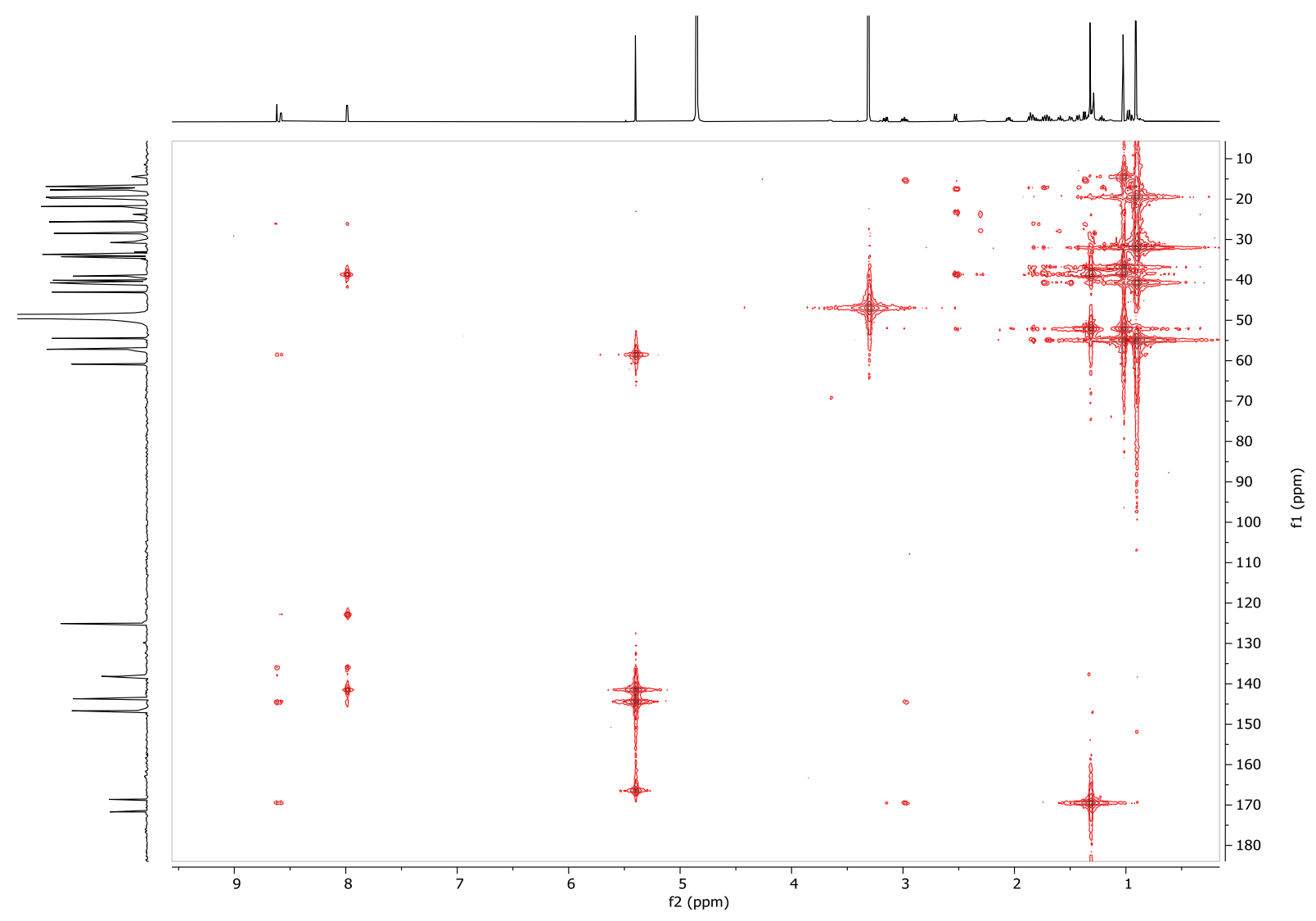




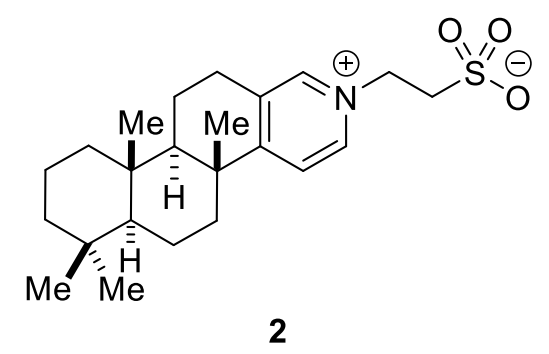

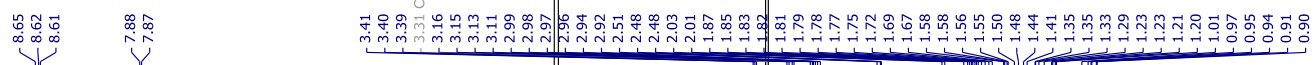

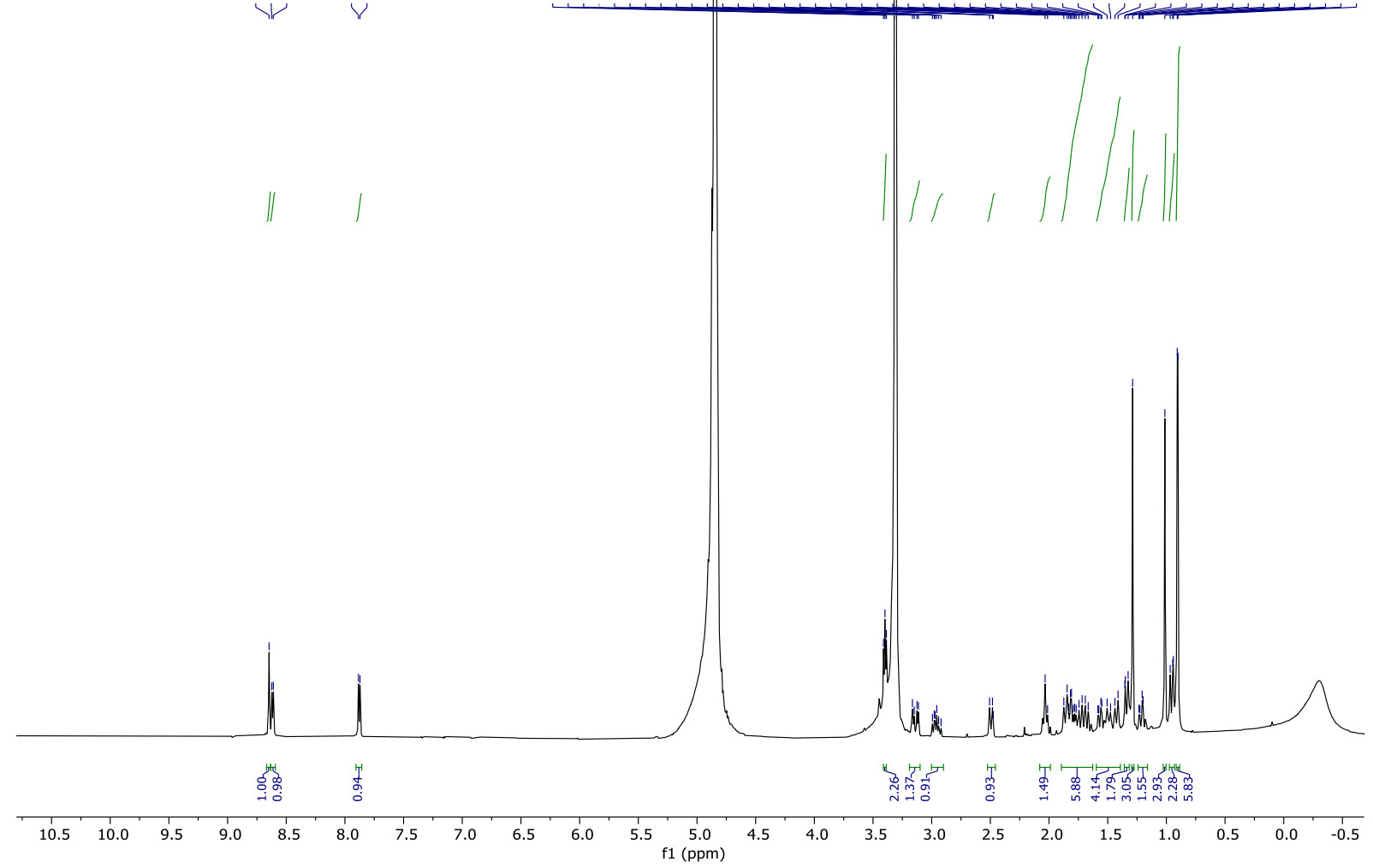



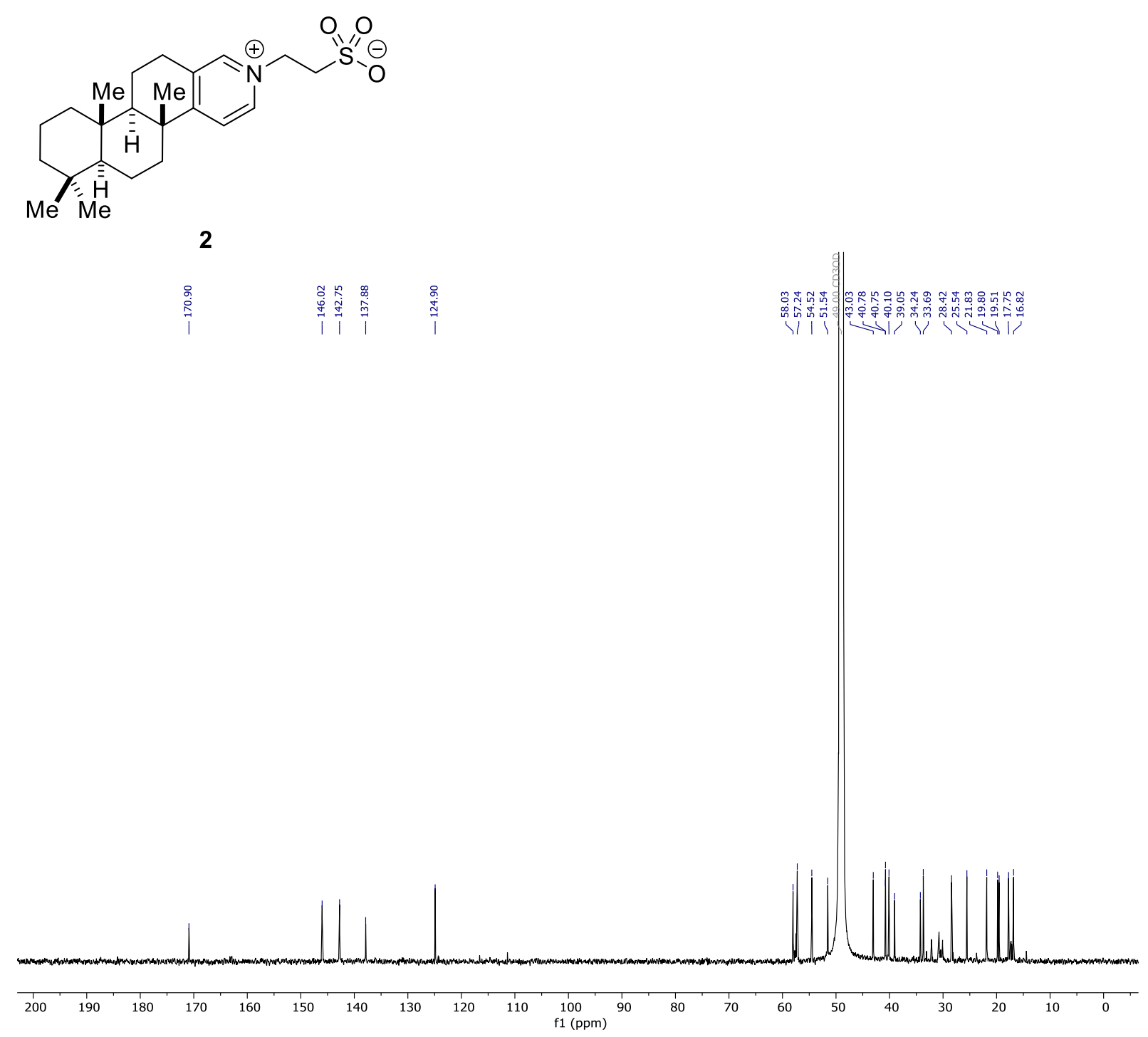
HMQC of spongidine D (2)

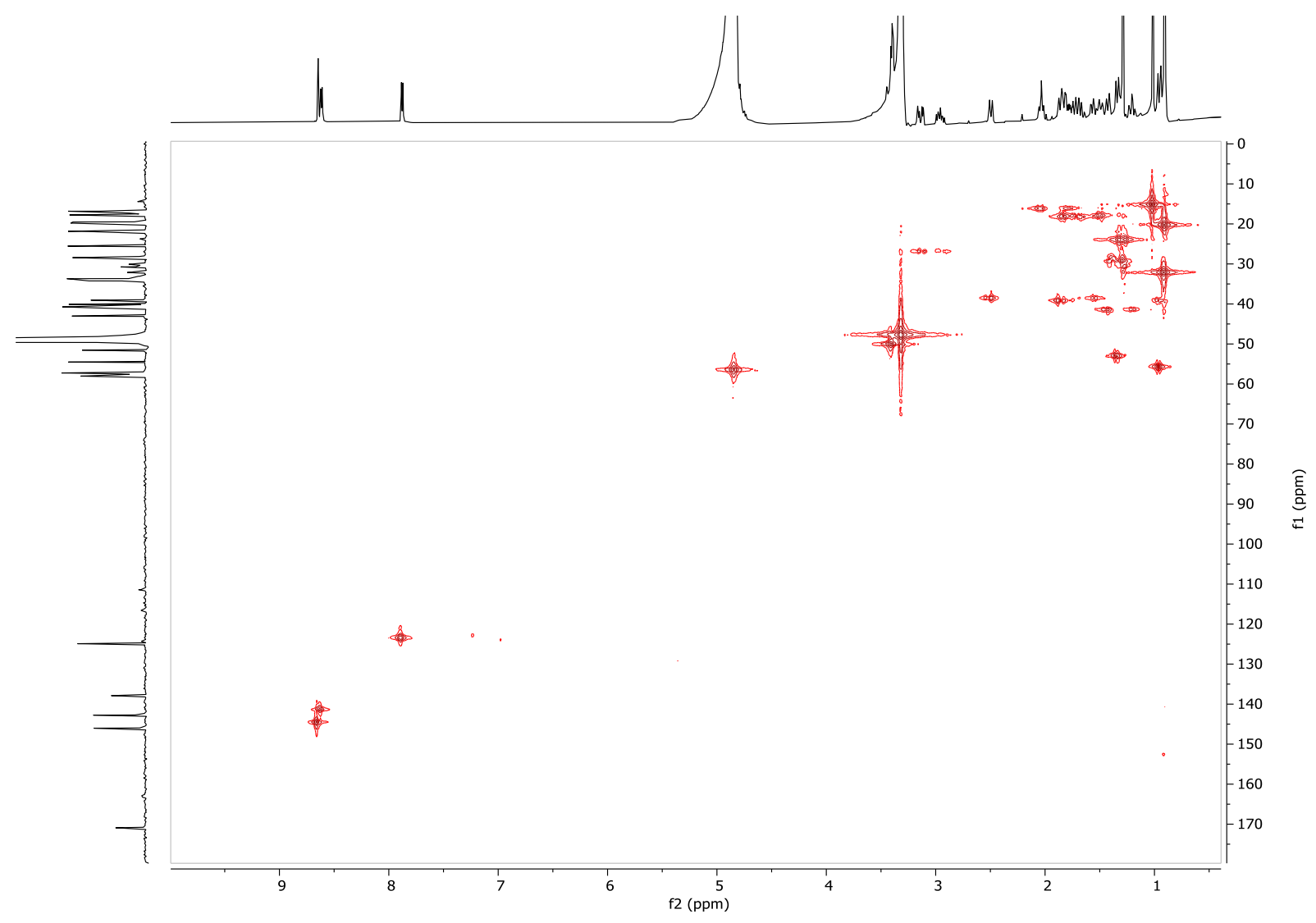


HMBC of spongidine D (2)

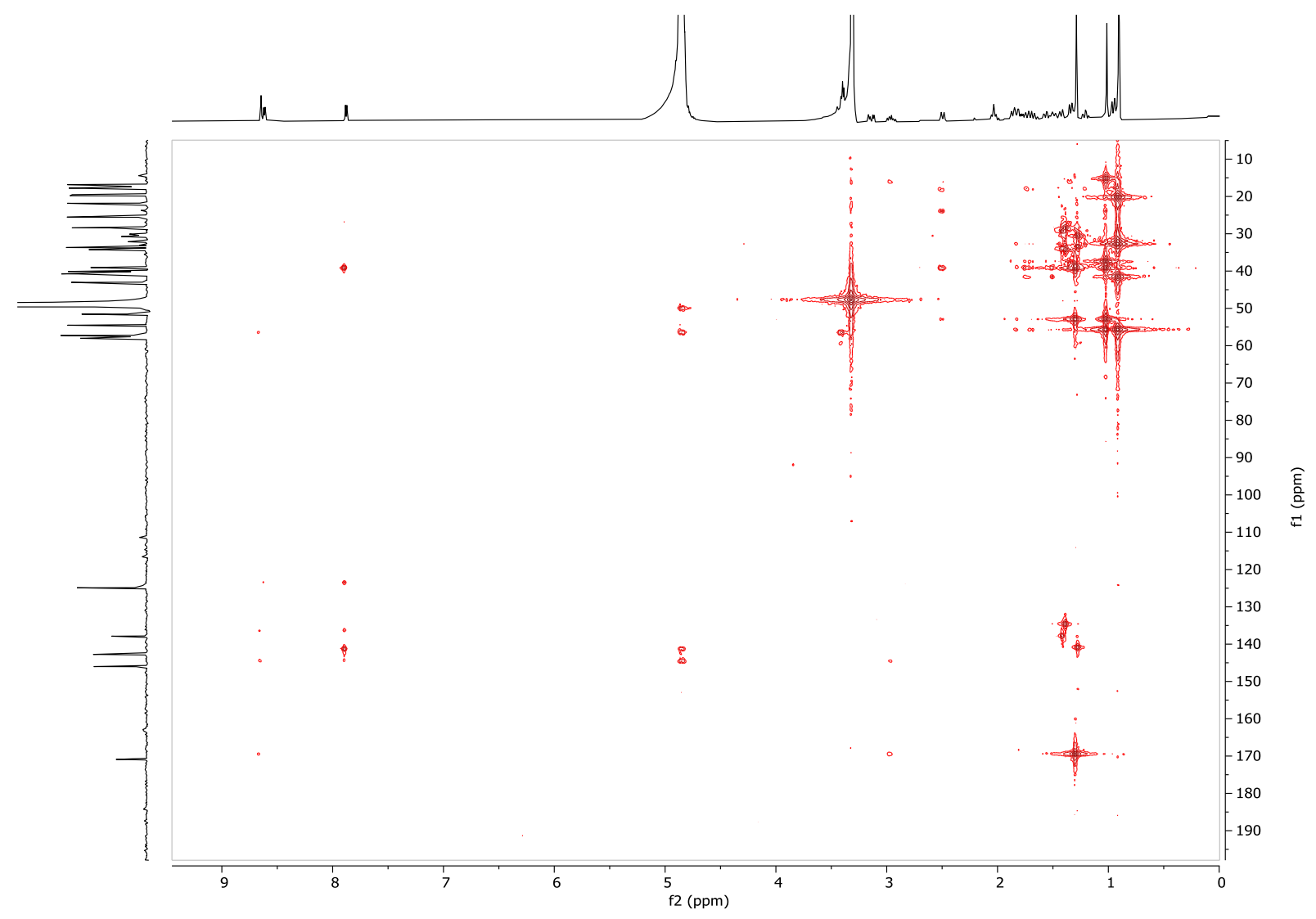



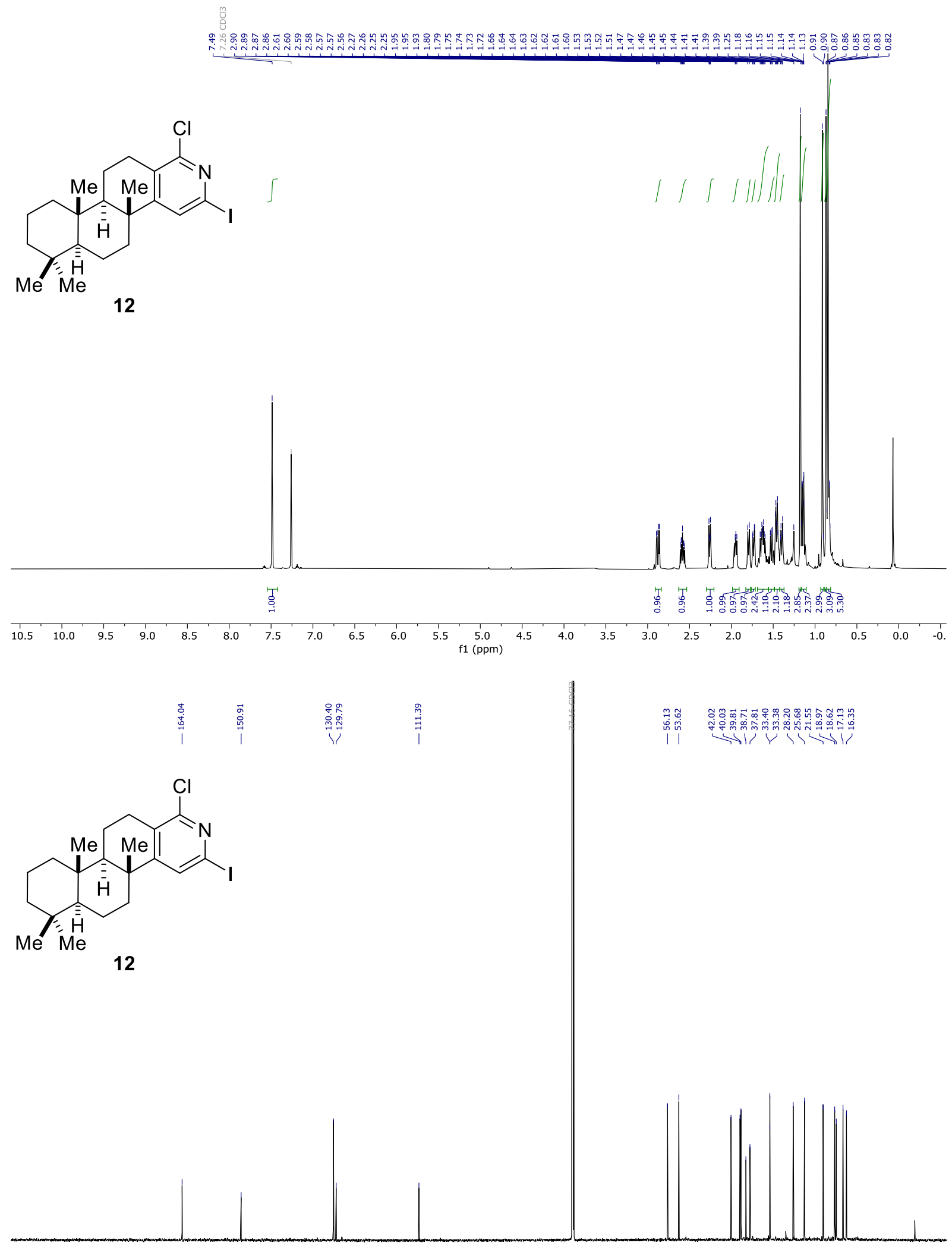

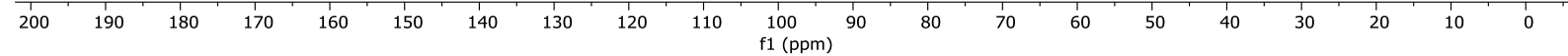




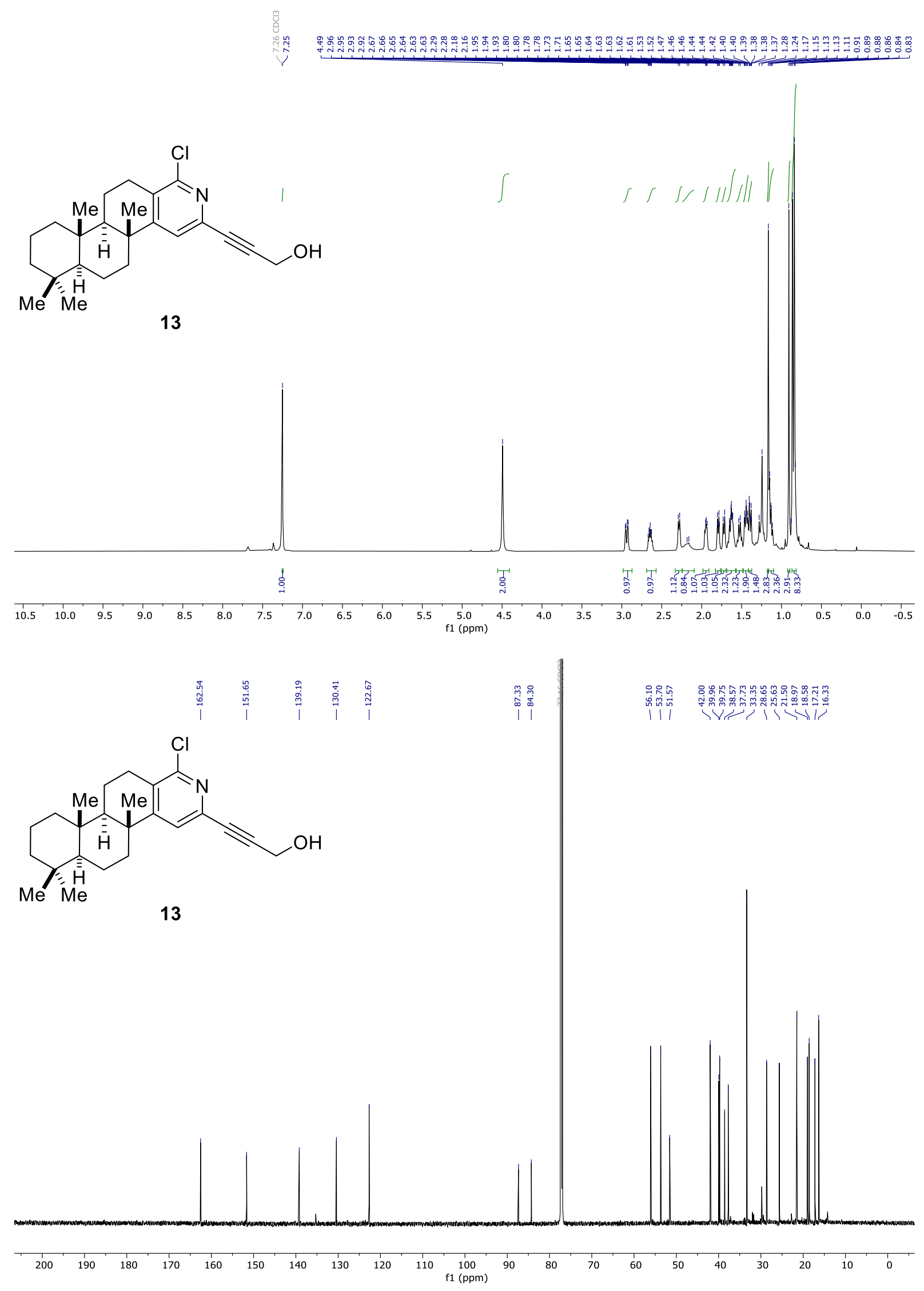



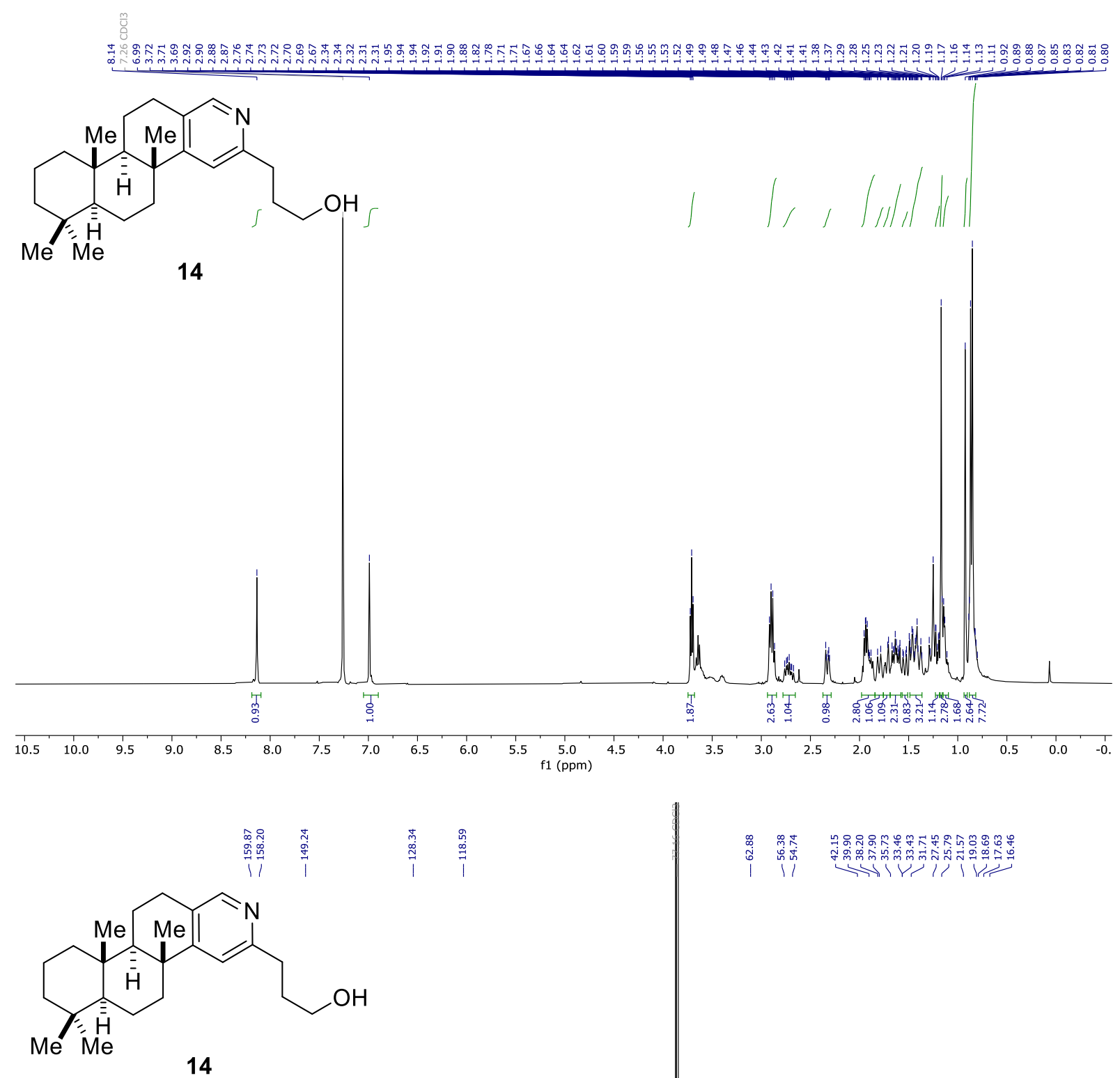

|

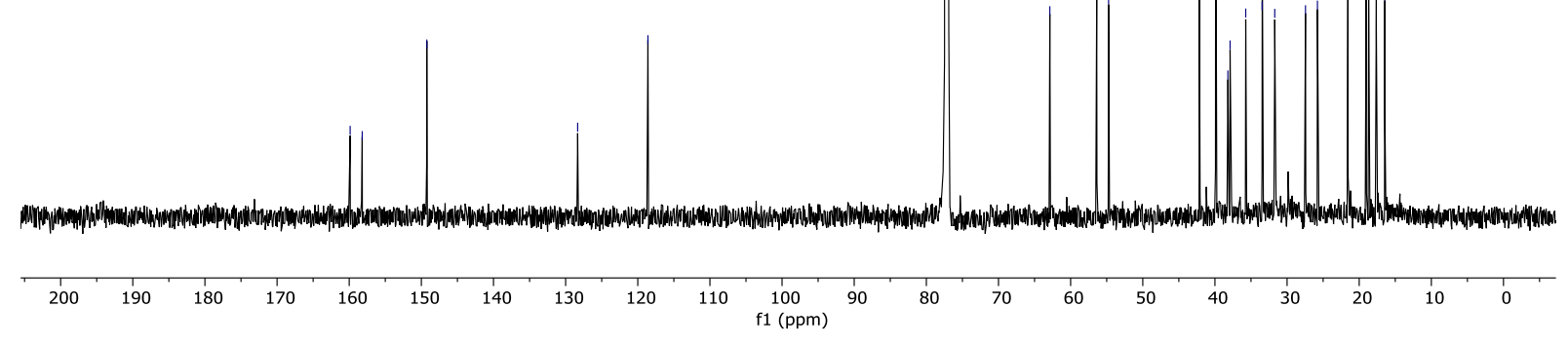


<smiles>[M]C12CCc3cnc(CCC(=O)OC)cc3C1(C)C1CCCC(C)(C)C1(C)CC2</smiles>

15

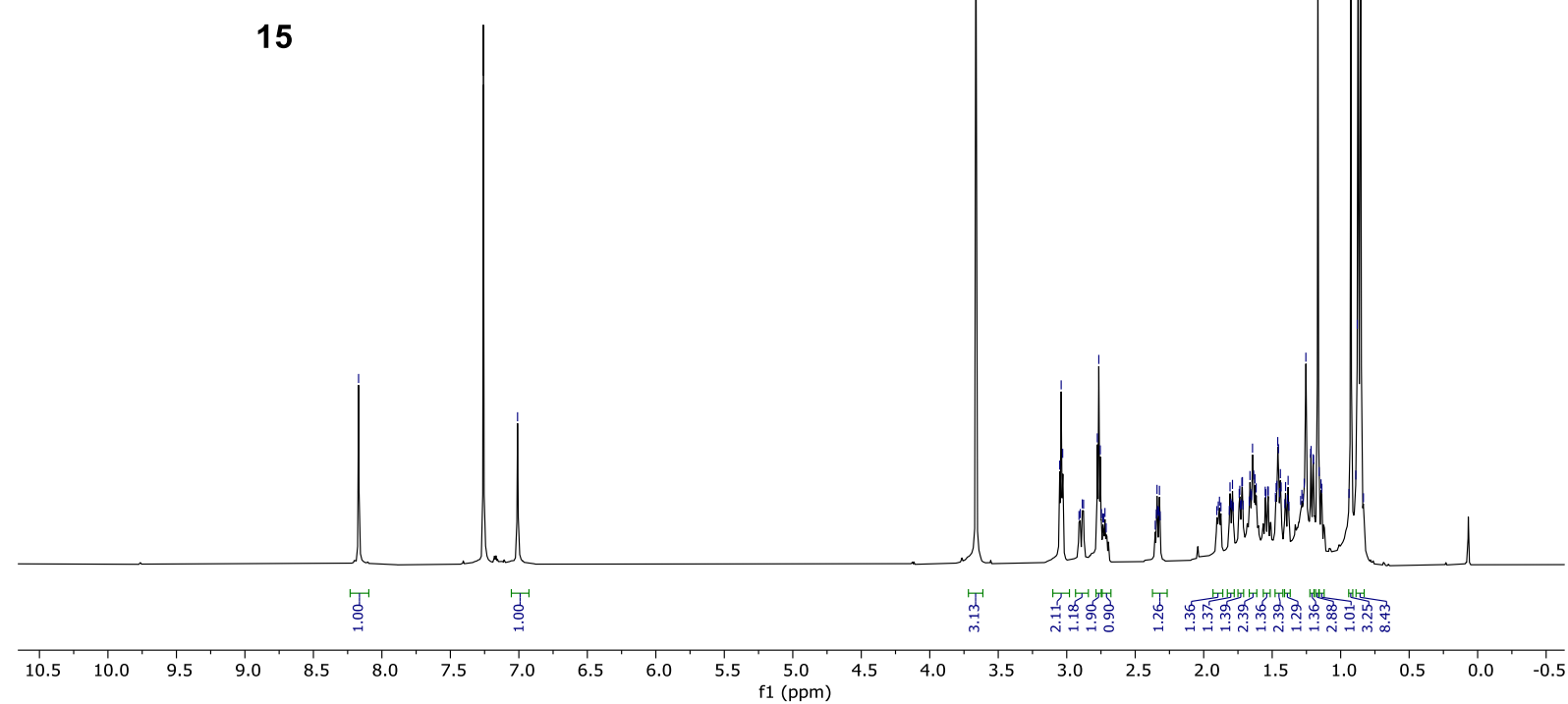

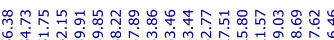

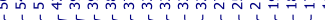<smiles>[M]C12CCCc3cnc(CCC(=O)OC)cc3C1C1CCCC(C)(C)C1(C)CC2</smiles>

15

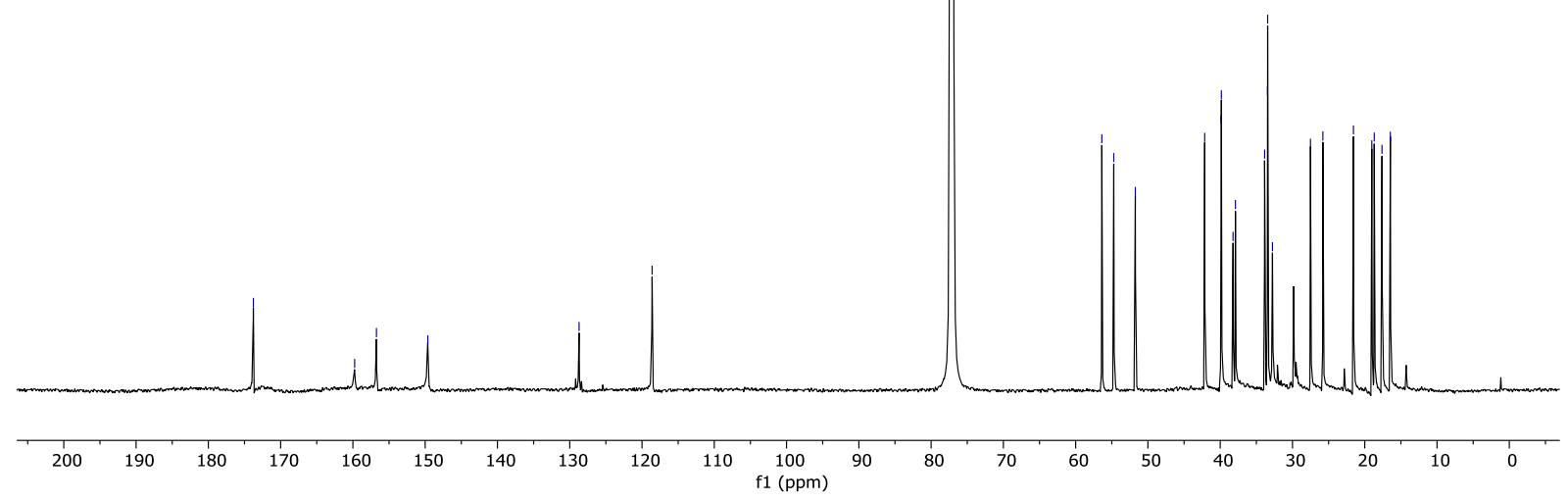


HMQC of petrosaspongiolide L methyl ester $\mathbf{1 5}$

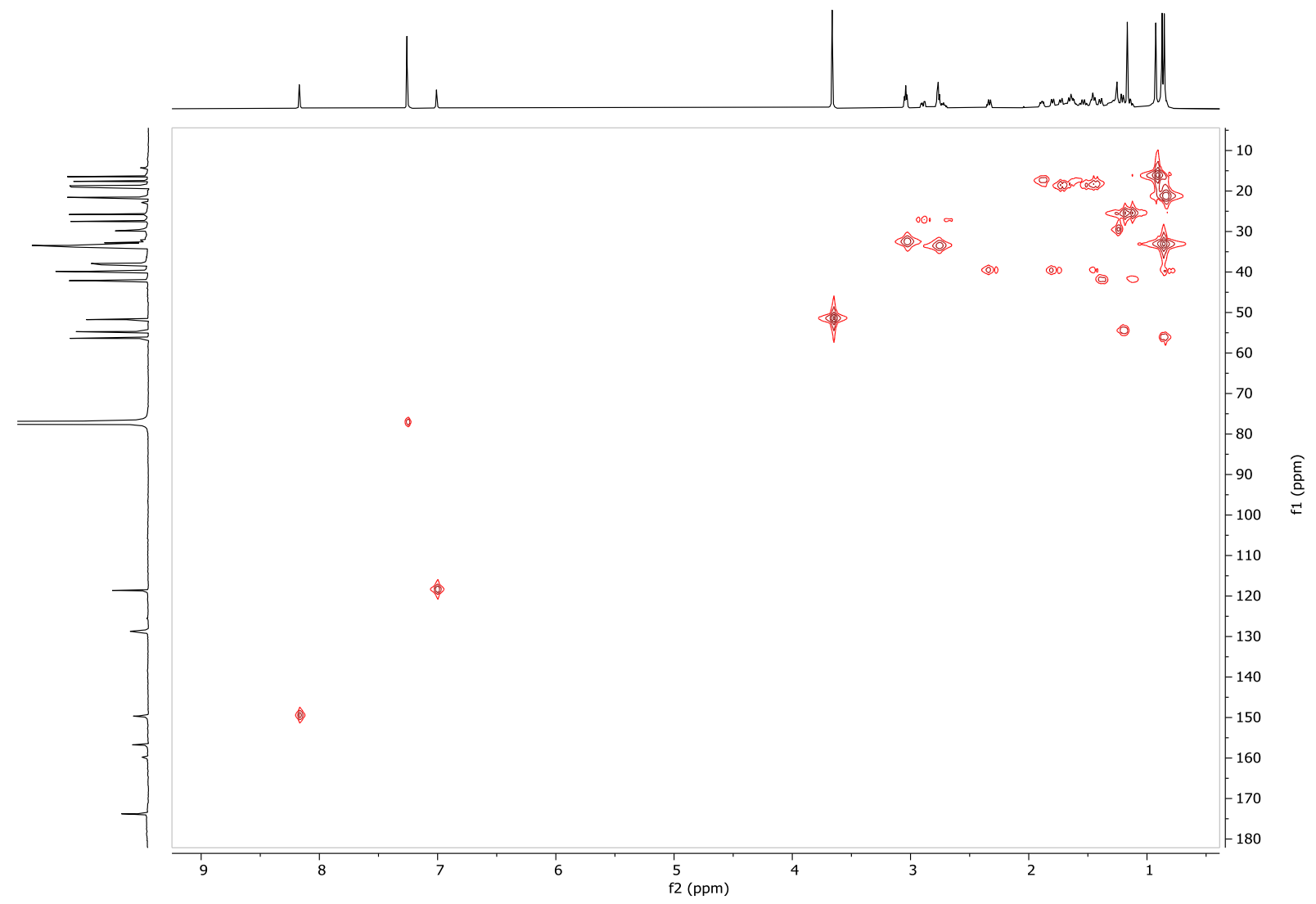


HMBC of petrosaspongiolide L methyl ester $\mathbf{1 5}$

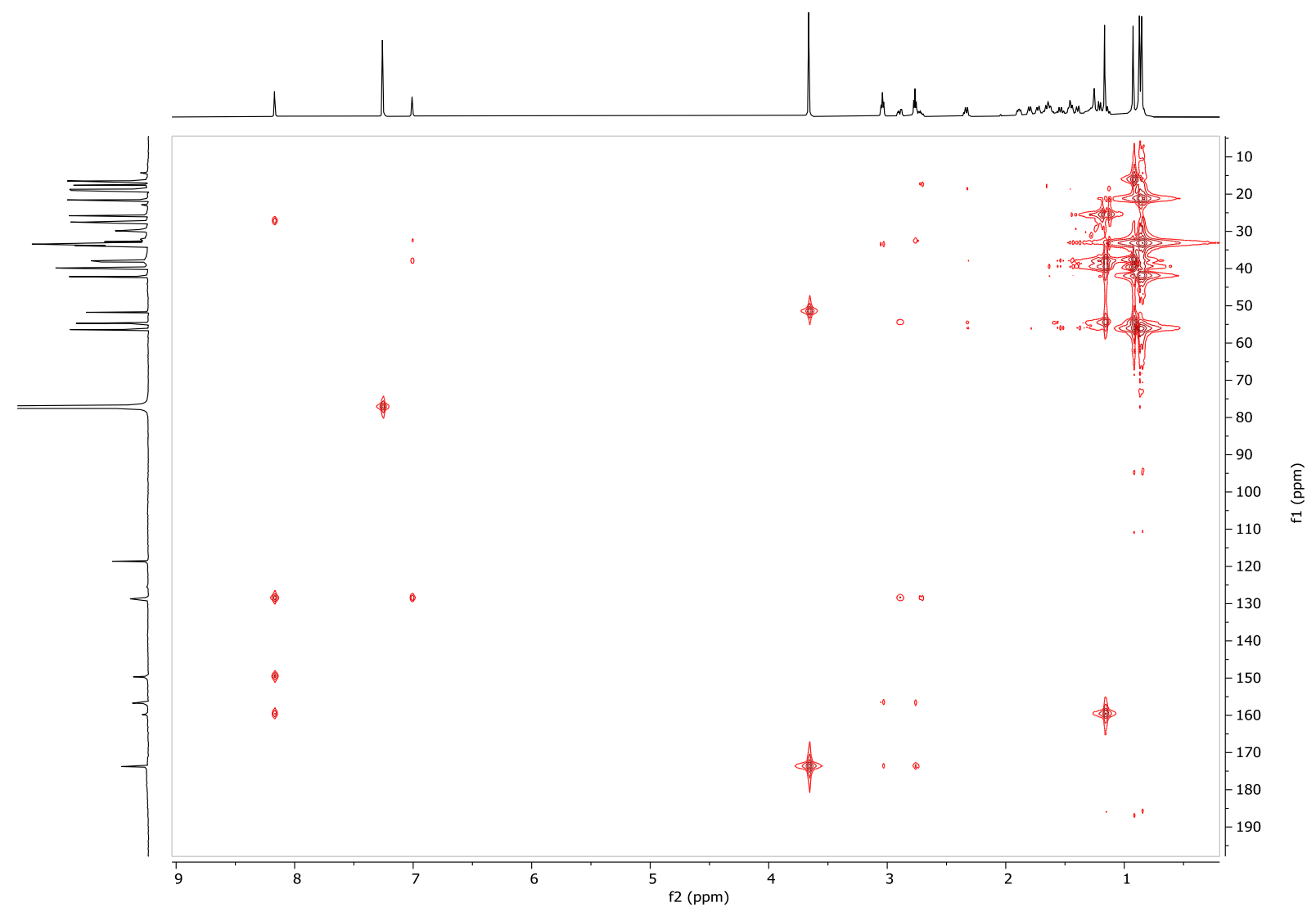


6. Crystallographic Data

Chloropyridine 4
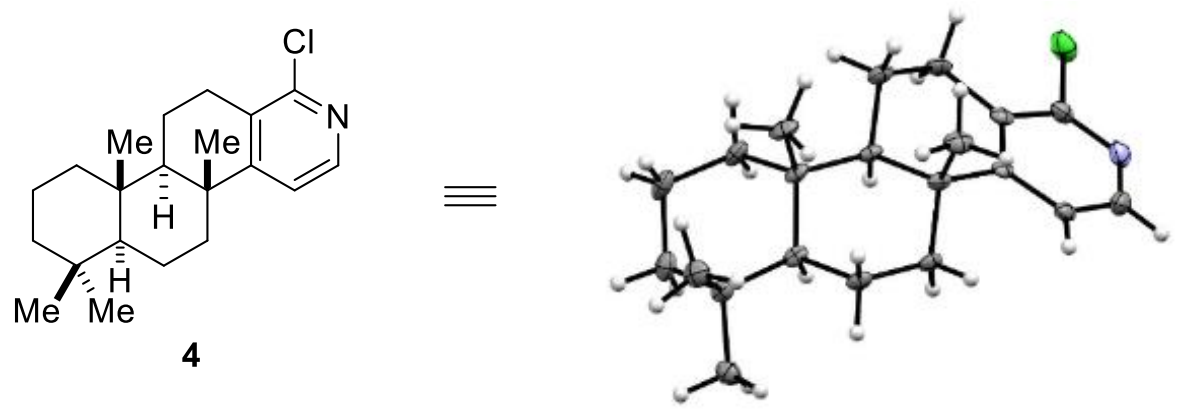

CCDC 1859575 contains the supplementary crystallographic data for this paper. These data can be obtained free of charge from The Cambridge Crystallographic Data Centre via www.ccdc.cam.ac.uk/structures 


\section{Previous Synthesis of Spongidine A and Spongidine D}

The synthesis of spongidine A and spongidine D by Basabe and coworkers was achieved in 2\% and $1 \%$ yield over 23 steps in the longest linear sequence, respectively. The synthetic effort was published in 3 publications (schemes SI2 to SI5, shown below for clarity). ${ }^{4,7,8}$ Additionally, the synthesis was published in the dissertation of Araceli Blanco Martín. ${ }^{6}$

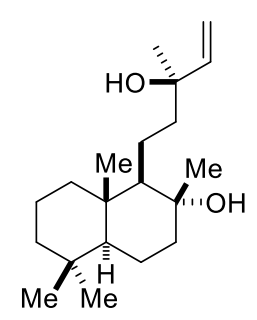

$(-)$-sclareol

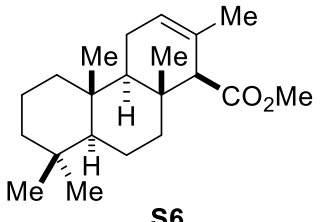

S6

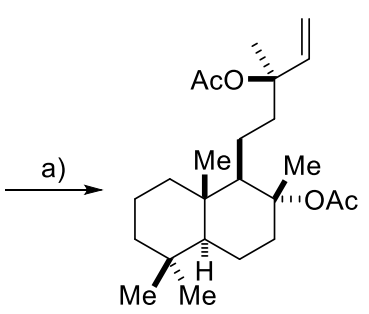

s2

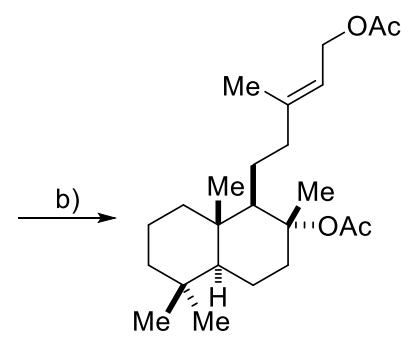

S3

Scheme SI2. Synthesis of ester S6, according to Basabe and co-workers. ${ }^{7}$ Reaction conditions: a) $\mathrm{AcCl}, \mathrm{CH}_{2} \mathrm{Cl}_{2}$, $N, N$-Dimethylanilin, rt, $12 \mathrm{~h}$, quant.; b) $\mathrm{PdCl}_{2}\left(\mathrm{MeCN}_{2}\right.$, THF, rt, $1 \mathrm{~h}, 92 \%$; c) $\mathrm{SiO}_{2}$, hexane, $100{ }^{\circ} \mathrm{C}, 1 \mathrm{~h}, 88 \%$; d) $\mathrm{K}_{2} \mathrm{CO}_{3}, \mathrm{MeOH}$, rt, 6 h, quant.; e) $\mathrm{MnO}_{2}, \mathrm{CH}_{2} \mathrm{Cl}_{2}, \mathrm{rt}, 8 \mathrm{~h}, 85 \%$; f) $\mathrm{NaClO}_{2}, \mathrm{NaH}_{2} \mathrm{PO}_{4}$, 2-methyl-2-butene, $t$-BuOH, rt, 12 h; g) $\mathrm{TMSCHN}_{2}, \mathrm{C}_{6} \mathrm{H}_{6} / \mathrm{MeOH}(1: 1), \mathrm{rt}, 10$ min, $89 \%$ over 2 steps; h) $\mathrm{HCOOH}, 65^{\circ} \mathrm{C}, 1.5 \mathrm{~h}, 89 \%$.

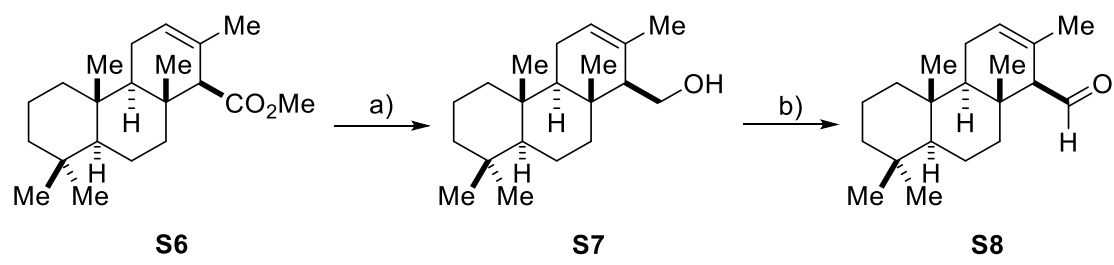

Scheme SI3. Synthesis of aldehyde S8, according to Basabe and co-workers. ${ }^{8}$ Reaction conditions: a) DIBAL-H, $\mathrm{CH}_{2} \mathrm{Cl}_{2},-78{ }^{\circ} \mathrm{C}, 2 \mathrm{~h}, 60 \%$; b) TPAP, NMO, $\mathrm{CH}_{2} \mathrm{Cl}_{2}$, MS $4 \stackrel{\AA}{\mathrm{A}}, \mathrm{rt}, 1 \mathrm{~h}, 98 \%$. 


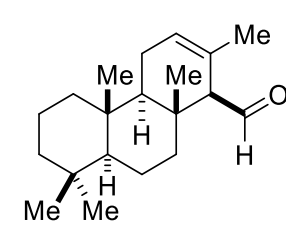

S8<smiles>CO/C=C/C1C(C)=CCC2C(C)C3(C)CCCC(C)(C)C3CCC12C</smiles>

S9<smiles>COC(=O)CC1C(C)=CCC2(C)C1(C)CCC1C(C)(C)CCCC1(C)C21CCCCC1</smiles>

e)<smiles>CCCOC(=O)CC1C(CCl)=CCCC1(C)C1CCC2C(C)(C)CCCC2(C)C1C</smiles>

S13

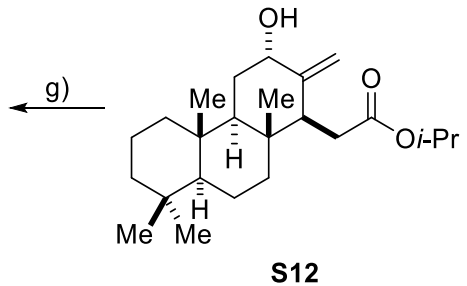

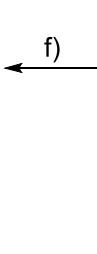

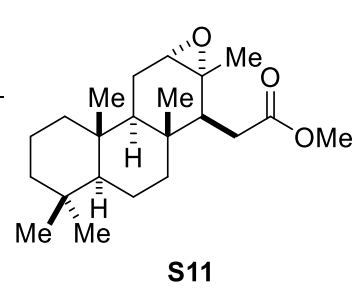

Scheme SI4. Synthesis of allyl chloride S13, according to Basabe and co-workers. ${ }^{4}$ Reaction conditions: a) $\mathrm{MeOCH}_{2} \mathrm{PPh}_{3} \mathrm{Cl}$, THF, NaHMDS, $-78{ }^{\circ} \mathrm{C}, 1 \mathrm{~h}, 80 \%$; b) $p$-TsOH, acetone, RT, $2 \mathrm{~h}, 99 \%$; c) $\mathrm{NaClO}_{2}, t$ - $\mathrm{BuOH}$, 2-methyl-2-butene, $\mathrm{NaH}_{2} \mathrm{PO}_{4}, \mathrm{rt}, 3 \mathrm{~h}$; d) $\mathrm{TMSCHN}_{2}, \mathrm{C}_{6} \mathrm{H}_{6} / \mathrm{MeOH} 1: 1,0{ }^{\circ} \mathrm{C}, 10 \mathrm{~min}, 85 \%$ over 2 steps; e) $m$-CPBA, $\mathrm{CH}_{2} \mathrm{Cl}_{2}, 0{ }^{\circ} \mathrm{C}$ to rt, $2 \mathrm{~h}, 98 \%$; f) $\mathrm{Al}(i \text {-PrO })_{3}$, toluene, $\left.150{ }^{\circ} \mathrm{C}, 16 \mathrm{~h}, 50 \% ; \mathrm{g}\right) \mathrm{SOCl}_{2}, \mathrm{Et}_{2} \mathrm{O}, 0{ }^{\circ} \mathrm{C}$ to rt, $3 \mathrm{~h}, 89 \%$.<smiles>CCCCCCC1CCC2(C)C(C)(C)CCCC2(C)C1CC(=O)OC(C)C</smiles>

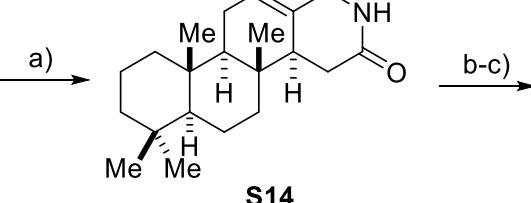<smiles>CC1(C)CCCC2(C)C1CCC1(C)c3cc(=O)[nH]cc3CCC12C</smiles>

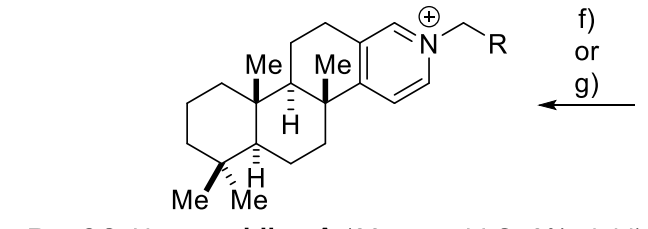

$\mathrm{R}=\mathrm{CO}_{2} \mathrm{H}$ spongidine $\mathrm{A}$ (23 steps LLS, $2 \%$ yield)

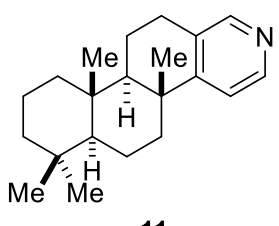

11

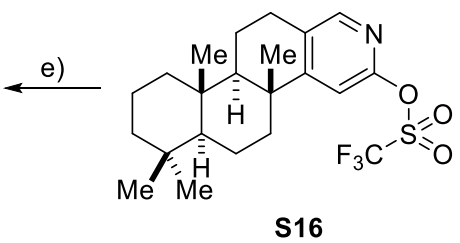

S16 $\mathrm{R}=\mathrm{CH}_{2} \mathrm{SO}_{3} \mathrm{H}$ spongidine $\mathbf{D}(23$ steps LLS, $1 \%$ yield $)$

Scheme SI5. Synthesis of spongidine A and spongidine D, according to Basabe and co-workers. ${ }^{4}$ Reaction conditions: a) $\mathrm{NH}_{4} \mathrm{OH}, \mathrm{EtOH}, 50{ }^{\circ} \mathrm{C}, 7 \mathrm{~h}, 73 \%$; b) $\mathrm{HI}, \mathrm{C}_{6} \mathrm{H}_{6}, 80{ }^{\circ} \mathrm{C}, 5 \mathrm{~h}, 99 \%$; c) LDA, THF, $\mathrm{O}_{2}$ (air), $-78{ }^{\circ} \mathrm{C}$ to $45^{\circ} \mathrm{C}, 5 \mathrm{~h}, 91 \%$; d) $\mathrm{Tf}_{2} \mathrm{O}, \mathrm{CH}_{2} \mathrm{Cl}_{2}$, pyridine, $-78^{\circ} \mathrm{C}$ to RT, $2 \mathrm{~h}, 66 \%$; e) $\mathrm{Pd}(\mathrm{OAc})_{2}, \mathrm{dppf} \mathrm{NEt}_{3}, \mathrm{NH}_{4}\left(\mathrm{HCO}_{2}\right), \mathrm{DMF}$, $60{ }^{\circ} \mathrm{C}, 3 \mathrm{~h}, 67 \%$; f) $\mathrm{BrCH}_{2} \mathrm{COOH}, \mathrm{C}_{6} \mathrm{H}_{5} \mathrm{Br}, 85^{\circ} \mathrm{C}, 24 \mathrm{~h}, 56 \%$; g) $\mathrm{BrCH}_{2} \mathrm{CH}_{2} \mathrm{SO}_{3} \mathrm{Na}, \mathrm{DMF}, 100{ }^{\circ} \mathrm{C}, 15 \mathrm{~h}, 49 \%$. 


\section{References}

(1) Bartels, F.; Hong, Y. J.; Ueda, D.; Weber, M.; Sato, T.; Tantillo, D. J.; Christmann, M. Chem. Sci. 2017, 8, 8285-8290.

(2) Kuethe, J. T.; Wong, A.; Davies, I. W. J. Org. Chem. 2004, 69, 7752-7754.

(3) Sakamoto, T.; Kondo, Y.; Yamanaka, H. Chem. Pharm. Bull. 1985, 33, 4764-4768.

(4) Basabe, P.; Blanco, A.; Marcos, I. S.; Díez, D.; Bodero, O.; Martín, M.; Urones, J. G. Tetrahedron 2011, 67, 3649-3658.

(5) De Marino, S.; Iorizzi, M.; Zollo, F.; Debitus, C.; Menou, J.-L.; Ospina, L. F.; Alcaraz, M. J.; Payá, M. J. Nat. Prod. 2000, 63, 322-326.

(6) Blanco-Martín, A. Síntesis de piridín alcaloides inhibidores de fosfolipasa A2: espongidinas. Dissertation, University of Salamanca, Salamanca, Spain, 2011.

(7) Urones, J. G.; Marcos, I. S.; Basabe, P.; Gomez, A.; Estrella, A.; Lithgow, A. M. Nat. Prod. Lett. 1994, 5, 217-220.

(8) Basabe, P.; Delgado, S.; Marcos, I. S.; Diez, D.; Diego, A.; De Román, M.; Urones, J. G. J. Org. Chem. 2005, 70, 9480-9485. 